\title{
Comprehensive Comparative Analysis of Cholesterol Catabolic Genes/Proteins in Mycobacterial Species
}

\author{
Rochelle van Wyk ${ }^{1}{ }^{\circledR}$, Mari van Wyk ${ }^{1}$, Samson Sitheni Mashele ${ }^{1}$, David R. Nelson ${ }^{2}(\mathbb{D}$ and \\ Khajamohiddin Syed ${ }^{3, *(1)}$ \\ 1 Unit for Drug Discovery Research, Department of Health Sciences, Faculty of Health and Environmental \\ Sciences, Central University of Technology, Bloemfontein 9300, Free State, South Africa; \\ rochellevanwyk0@gmail.com (R.v.W.); marivanwyk764@gmail.com (M.v.W.); smashele@cut.ac.za (S.S.M.) \\ 2 Department of Microbiology, Immunology and Biochemistry, University of Tennessee Health Science Center, \\ Memphis, TN 38163, USA; drnelson1@gmail.com \\ 3 Department of Biochemistry and Microbiology, Faculty of Science and Agriculture, University of Zululand, \\ KwaDlangezwa 3886, South Africa \\ * Correspondence: khajamohiddinsyed@gmail.com; Tel.: +27-0359026875
}

Received: 21 January 2019; Accepted: 12 February 2019; Published: 27 February 2019

\begin{abstract}
In dealing with Mycobacterium tuberculosis, the causative agent of the deadliest human disease-tuberculosis (TB) - utilization of cholesterol as a carbon source indicates the possibility of using cholesterol catabolic genes/proteins as novel drug targets. However, studies on cholesterol catabolism in mycobacterial species are scarce, and the number of mycobacterial species utilizing cholesterol as a carbon source is unknown. The availability of a large number of mycobacterial species' genomic data affords an opportunity to explore and predict mycobacterial species' ability to utilize cholesterol employing in silico methods. In this study, comprehensive comparative analysis of cholesterol catabolic genes/proteins in 93 mycobacterial species was achieved by deducing a comprehensive cholesterol catabolic pathway, developing a software tool for extracting homologous protein data and using protein structure and functional data. Based on the presence of cholesterol catabolic homologous proteins proven or predicted to be either essential or specifically required for the growth of M. tuberculosis H37Rv on cholesterol, we predict that among 93 mycobacterial species, 51 species will be able to utilize cholesterol as a carbon source. This study's predictions need further experimental validation and the results should be taken as a source of information on cholesterol catabolism and genes/proteins involved in this process among mycobacterial species.
\end{abstract}

Keywords: Cholesterol catabolism; Cholesterol catabolic genes/proteins; Comparative analysis; in silico analysis; Mycobacterium tuberculosis; Mycobacterium tuberculosis complex; Tuberculosis; Mycobacterium chelonae-abscessus complex; Mycobacterium avium complex; Mycobacteria causing leprosy; Non-tuberculous mycobacteria; Saprophytes; Software tool

\section{Introduction}

Tuberculosis (TB), is a chronic infectious disease caused by Mycobacterium tuberculosis, and is one of the leading causes of death worldwide, killing an estimated two million people annually $[1,2]$. It is estimated that one third of the world's population (approximately two billion people) is infected with this highly pathogenic organism [3]. Once it has entered the human body, and after ingestion by macrophages, this intracellular pathogen can survive in a modified phagosome and cause latent infection for years and sometimes decades without any symptoms [4]. Tubercle bacilli can persist in this dormant state, from which they may be reactivated and cause TB [4]. The reactivation of latent phase M. tuberculosis into the active phase is observed among people whose immune systems are weakened 
by HIV infection, by immunosuppressive drugs or by malnutrition and/or aging [5]. Over the past decades, the threat of TB has become greater with the development of single-drug resistance to multiple-drug-resistant strains and, recently, the surfacing of extensive drug resistance that threatens to compromise the available drugs severely [6]. With the documentation of total drug-resistant strains [6], along with the insufficiency of new drug targets, we clearly need more research to discover novel drug targets.

M. tuberculosis can infect, grow and survive in the harsh environment of the macrophage and other host cells using mechanisms that are not yet well understood $[7,8]$. Host cholesterol levels are thought to play a role in the development of $M$. tuberculosis infection [9], with high levels of cholesterol in the diet significantly enhancing the bacterial burden in the lung [10] and impairing immunity to M. tuberculosis [11]. Specifically, cholesterol is required for the phagocytosis of mycobacteria into macrophages [12,13], where they bind and enter phagocytes through cholesterol-enriched membrane microdomains (lipid rafts) [14]. In addition, cholesterol plays a crucial role in the mediation of the infected phagosomal association of tryptophan-aspartate-containing coat protein [15], leading to the inhibition of phagosome-lysosome fusion [16]. This experimental evidence suggests an important role for cholesterol during $M$. tuberculosis infection and persistence.

Research studies have demonstrated that $M$. tuberculosis can grow using cholesterol as the sole carbon and energy source [17]. Therefore, cholesterol has recently been identified as an important lipid for mycobacterial infection $[18,19]$. The relatively abundant cholesterol distributed in host cells is an important growth substrate for these bacteria in different infection stages (e.g., intracellular growth or intracellular persistence) [20]. M. tuberculosis growing in human cells appears to obtain energy from host lipids rather than other nutrients such as carbohydrates [21].

Considering the above facts and recent momentum on cholesterol catabolism as a therapeutic target in M. tuberculosis, Ouellet and co-workers [19] suggest that more research needs to be done to understand cholesterol catabolism in mycobacterial species. Furthermore, performing laboratory experiments is laborious and time- and money-consuming, since each mycobacterial species has a different lifestyle and different culture conditions. Taking advantage of the genome sequencing of many mycobacterial species, this study is aimed at performing comprehensive comparative analysis of the genes/proteins involved in cholesterol catabolism and predicting mycobacterial species' ability to utilize cholesterol as a carbon source.

\section{Results and Discussion}

\subsection{Deducing Cholesterol Catabolic Pathway in M. Tuberculosis H37Rv}

Based on the available literature [19,22-27], the cholesterol catabolic pathway in M. tuberculosis can be divided into two major phases - the initial degradation of the aliphatic side chain (Figure 1) and the subsequent degradation of the four alicyclic A-D rings (Figures 2 and 3). It has not been confirmed whether there is a specific order to the degradation reactions regarding the side chain and rings, but for $M$. tuberculosis it has been suggested that the ring-degrading enzymes KsaAB and HsaA-C act optimally after the side chain has been removed, since blockage of the side chain degradation resulted in accumulation of cholest-4-en-3-one as a major metabolite [19].

\subsubsection{Degradation of Cholesterol: Side Chain Degradation}

It is generally accepted that the cholesterol side chain is shortened by $\beta$-oxidation reactions [19]. Before the saturated side chain of cholesterol can enter into the $M$. tuberculosis $\beta$-oxidation pathway, it must first be chemically functionalized at the $\omega$-position [19] (Figure 1). Of the four chemical steps necessary to prepare the side chain for $\beta$-oxidation, the first three are oxidation reactions catalyzed by cytochrome P450 enzymes CYP125 (Rv3545c), CYP142 (Rv3518c) and CYP124 (Rv2266) [19,28]. These are capable of oxidizing the side chains of cholesterol and cholest-4-en-3-one to the terminal alcohol 
(by introducing a hydroxyl group onto the side chain), aldehyde and carboxylic acid metabolites. A sterol-CoA ligase catalyzes the final ATP-dependent step [19] (Figure 1).

Research has demonstrated that CYP125 does not play a key role in cholesterol catabolism in the M. tuberculosis H37Rv strain and suggests that this strain carries out compensatory activities [29]. However, investigation of the in vitro enzyme specificities found that CYP125 and CYP142 are the dominant P450 enzymes responsible for initiating sterol side chain degradation in M. tuberculosis [29], although in the CDC1551 strain, CYP142 is present as a pseudogene [30]. In vitro analysis has also demonstrated that CYP142 can support the growth of the H37Rv strain on cholesterol in the absence of cyp125A1 [29]. Using western blot analysis, researchers found that CYP124A1 was not detectably expressed in the H37Rv or CDC1551 strains, but CYP142 was found in H37Rv and not in CDC1551 [29]. In the absence of CYP125 or CYP142, cholest-4-en-3-one accumulates and inhibits bacterial growth on cholesterol [19].

$\beta$-oxidation is the pathway of the breakdown of fatty acids in the form of acyl-CoA molecules, [24]. Before the oxidative reactions of the $\beta$-oxidation cycle, the fatty acid is activated in a reaction catalyzed by an ATP-dependent ligase, to its thioester with coenzyme A (CoA). The thioester then undergoes dehydrogenation catalyzed by acyl-CoA dehydrogenase to form the enoyl-CoA, which is then hydrated to the hydroxyacyl-CoA by enoyl-CoA hydratase. Next, 3-hydroxyacyl-CoA dehydrogenase catalyzes the oxidation of the hydroxyl group. The thiolase in the next step, carryout the thiolytic cleavage of $\beta$-ketoacyl-CoA into two molecules of acyl-CoA as products, seems to correspond to the FadA5. A single round of the $\beta$-oxidation cycle of unbranched chain fatty acids produces acetyl-CoA and a CoA thioester of an acid that is shorter by two carbon atoms. The shortened fatty acyl-CoA then undergoes a further round of the $\beta$-oxidation cycle [24].

Genes believed to be encoding $\beta$-oxidation enzymes have been identified in the cholesterol regulons of $M$. tuberculosis [19]. One of these enzymes, a thiolase encoded by fadA5, catalyzes the thiolysis of acetoacetyl-CoA in vitro, which is consistent with removal of the side chain by $\beta$-oxidation, producing androstene metabolites, 4 -androstenedione (AD) and 1,4-androstenedione (ADD). This activity is required for growth on cholesterol and virulence, especially during the late (chronic) stage of mouse infection, prior to the onset of the immune response [22,30]. Another set of enzymes, acyl-CoA dehydrogenases, is required to catalyze unsaturation reactions in $\beta$-oxidation of steroid-CoA substrates, and the $M$. tuberculosis genome contains six sets of these enzyme genes ( $\left.f a d E^{\prime} \mathrm{s}\right)$. Regulated by cholesterol, each set of these genes is found adjacent to another within the same operon [31].

The research of Schappinger et al. [32] indicates the induction of 18 genes predicted to encode all the enzymes necessary for the biochemical activation and $\beta$-oxidation of fatty acids, including fatty acid-CoA synthase (fadD3, fadD9, fadD10, fadD19), acyl-CoA dehydrogenase (fadE5, fadE14, fadE22-24, fadE27-29, fadE31), enoyl-CoA hydratase (echA19), hydroxybutyryl-CoA dehydrogenase (fadB2, fadB3) and acetyl-CoA transferase ( $f a d A 5, f a d A 6)$.

Griffin et al. [26] also found that $h s d 4 A$, another predicted $\beta$-oxidation gene, was required for growth on cholesterol, along with ltp2, fadE29, fadE28, fadA5, fadE30, fadE32, fadE33, fadE34, hsd4B and also fadE5, echA9, fadD36 and fadE25.

\subsubsection{Degradation of Cholesterol: Sterol Ring Degradation}

The first step in the breakdown of the sterol ring is the conversion of cholesterol to cholest-4-en-3-one (Figure 1). This reaction is catalyzed by either a $3 \beta$-HSD or a cholesterol oxidase (ChoD). As mentioned earlier, Rv1106c encodes a $3 \beta-H S D$. This enzyme uses NAD+ as a cofactor and oxidizes cholesterol (among others) to its 3-keto-4-ene product, cholest-4-en-3-one [19]. Rv3409c encodes $\mathrm{ChoD}$ and is required for M. tuberculosis virulence [33]. However, in a study by Yang et al. [34] it was found that $R v 3409 c$ was not required for growth on cholesterol as a sole carbon source, and they concluded that $3 \beta$-HSD is required for the initial conversion of cholesterol and that a second ChoD activity is not present in $M$. tuberculosis. In addition to this, mice infection experiments confirmed 
the significance of ChoD in the pathogenesis of M. tuberculosis, where it drives the oxidation of $3 \beta$-hydroxy-5-ene to 3-keto-4-ene [33].

It is assumed that 3-ketosteroid- $\Delta^{1}$-dehydrogenase $\left(\Delta^{1} \mathrm{KstD}\right)$ is coded by the $R v 3537$ gene that is part of the cholesterol regulon $[19,25]$. This enzyme catalyzes the trans-axial elimination of the $C 1(\alpha)$ and $\mathrm{C} 2(\beta)$ hydrogen atoms (C1-C2 dehydrogenation) of the 3-ketosteroid A ring of 4-androstenedione (AD) to yield 1,4-androstenedione (ADD) (Figure 2) [19], and targeted disruption of this gene inhibited growth on cholesterol [35]. In research done by Brzostek et al. [35], direct evidence was found that $M$. tuberculosis degrades cholesterol exclusively via the AD/ADD intermediates, and that KstD plays an essential role in this process.

In the next step, 9-hydroxylation of the 3-ketosteroid is catalyzed by KshAB (3-ketosteroid $9 \alpha$-hydroxylase), a two-component Rieske oxygenase, where KshA (Rv3526) is the oxygenase component and KshB (Rv3571) is the reductase component [36] (Figure 2). Research has shown that $\Delta k s h A$ and $\Delta k s h B$ deletion mutants are unable to utilize cholesterol and are essential in M. tuberculosis pathogenicity [37].

These two steps-the 9-hydroxylation of the 3-ketosteroid together with the C1-C2 dehydrogenation-are key to opening of the $\mathrm{B}$ ring and aromatization of the $\mathrm{A}$ ring via 9-hydroxy-1,4-androstene-3,17-dione (9OHADD) [19]. This intermediate is unstable and spontaneously hydrolyses to 3-hydroxy-9,10-secoandrosta-1,3,5(10)-triene-9,17-dione (3-HSA) [36].

The $h s a A C D B$ genes in $M$. tuberculosis are part of a single operon and transposon mutagenesis studies have indicated that their activity is critical for the survival of $M$. tuberculosis in macrophages [38,39]. The $h s a A$ and $h s a B$ genes encode for the putative oxygenase and reductase, respectively, of a flavin-dependent mono-oxygenase that hydroxylates (C4-hydroxylation) 3-HAS, a phenol, to a catechol, 3,4-dihydroxy-9,10-secoandrosta-1,3,5(10)-triene-9,17-dione (3,4-DHSA) [39]. Next, 3,4-DHSA is oxygenated and cleaved by $\mathrm{HsaC}$, an iron-dependent extradiol dioxygenase, to produce 4,5-9,10-diseco-3-hydroxy-5,9,17-trioxoandrosta-1(10),2-dien-4-oic acid (4,9-DSHA) [19]. The inactivation of $\mathrm{HsaC}$ results in the death of M. tuberculosis due to the accumulation of catechol metabolites [19]. HsaD, a member of the $\alpha / \beta$ hydrolase family, is involved in the aerobic degradation of aromatic compounds in microbes and is coded by $h s a D$, one of the genes identified as required for survival in macrophages [19]. HsaD is hypothesized to catalyze the hydrolysis of a carbon-carbon bond in 4,9-DSHA to yield 9,17-dioxo-1,2,3,4,10,19-hexanorandrostan-5-oic acid (DOHNAA) and 2-hydroxy-hexa-2,4-dienoic acid (HHD). HHD is then metabolized to tricarboxylic acid cycle intermediates [40] and propionyl-CoA [19], probably by HsaEFG (hsaEFG) [26]. The metabolic fate of DOHNAA (corresponding to the $C$ and D ring fragments), meanwhile, has recently been elucidated by Crowe et al. [27], who proposed a pathway for the metabolic fate of the $C$ and $\mathrm{D}$ rings of steroids (Figure 3). The proposal was that the last two steroid rings of DOHNAA (referred as HIP) are hydrolytically opened by enzymes encoded by the KstR2 regulon, where cleavage of ring $\mathrm{D}$ precedes that of ring $\mathrm{C}$ (Figure 3). The process is initiated by the degradation of the propionyl side chain by $\beta$-oxidation to yield $5-\mathrm{OH}$ HIP-CoA, which is then converted to HIEC-CoA ((7aS)-7a-methyl-1,5-dioxo-2,3,5,6,7,7a-hexahydro- $1 H$-indene-4-carboxyl-CoA) by IpdF and IpdC. The two consecutive ring cleavage reactions occur, where EchA20 catalyzes the hydrolysis of ring $\mathrm{D}$, followed by the hydrolysis of ring $C$ catalyzed by IpdAB. The metabolite resulting from the opened ring $C$ is then potentially thiolyzed by FadA6, or another thiolase, to produce MOODA-CoA. An acyl-CoA dehydrogenase, consisting wholly or partly of FadE32, then oxidizes this product to ${ }^{2} \Delta$-MOODA-CoA (4-methyl-5-oxo-octanedioicacid). It is proposed that a final round of $\beta$-oxidation yields 2-methyl- $\beta$-ketoadipyl-CoA (M $\beta$ KA-CoA), which can then be cleaved to produce propionyl-CoA and succinyl-CoA (Figure 3). Griffin et al. [26] identified genes fadE28, fadE29 and fadD3 to be probably involved in the degradation of DOHNAA. 


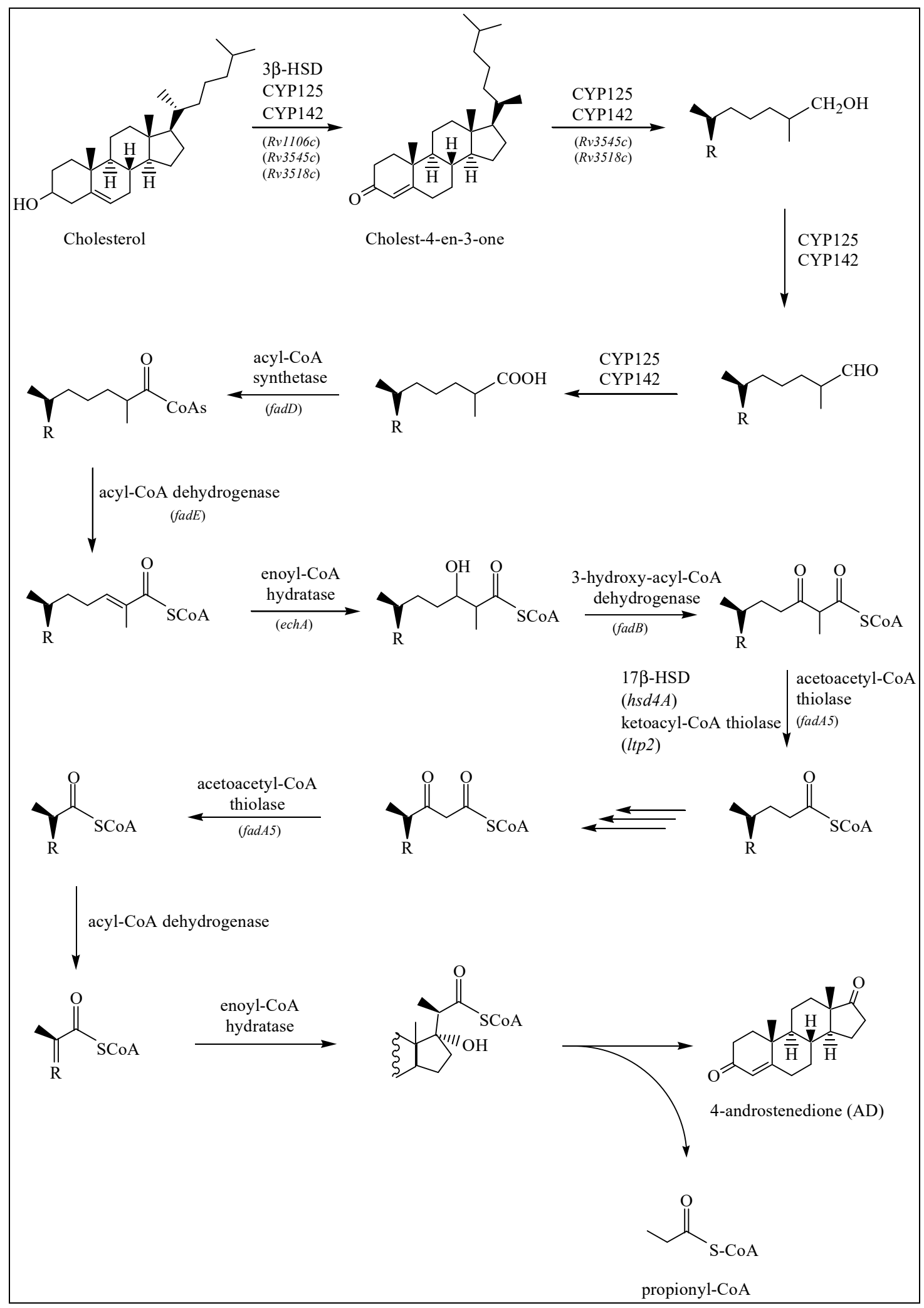

Figure 1. Cholesterol side chain degradation as described in Section 2.1.1. If known, the enzymes involved in each reaction are depicted by arrows, along with the gene coding for the specific enzyme. 




Figure 2. Cholesterol ring degradation as described in Section 2.1.2. If known, the enzymes involved in each reaction are depicted by arrows, along with the gene coding for the specific enzyme. 


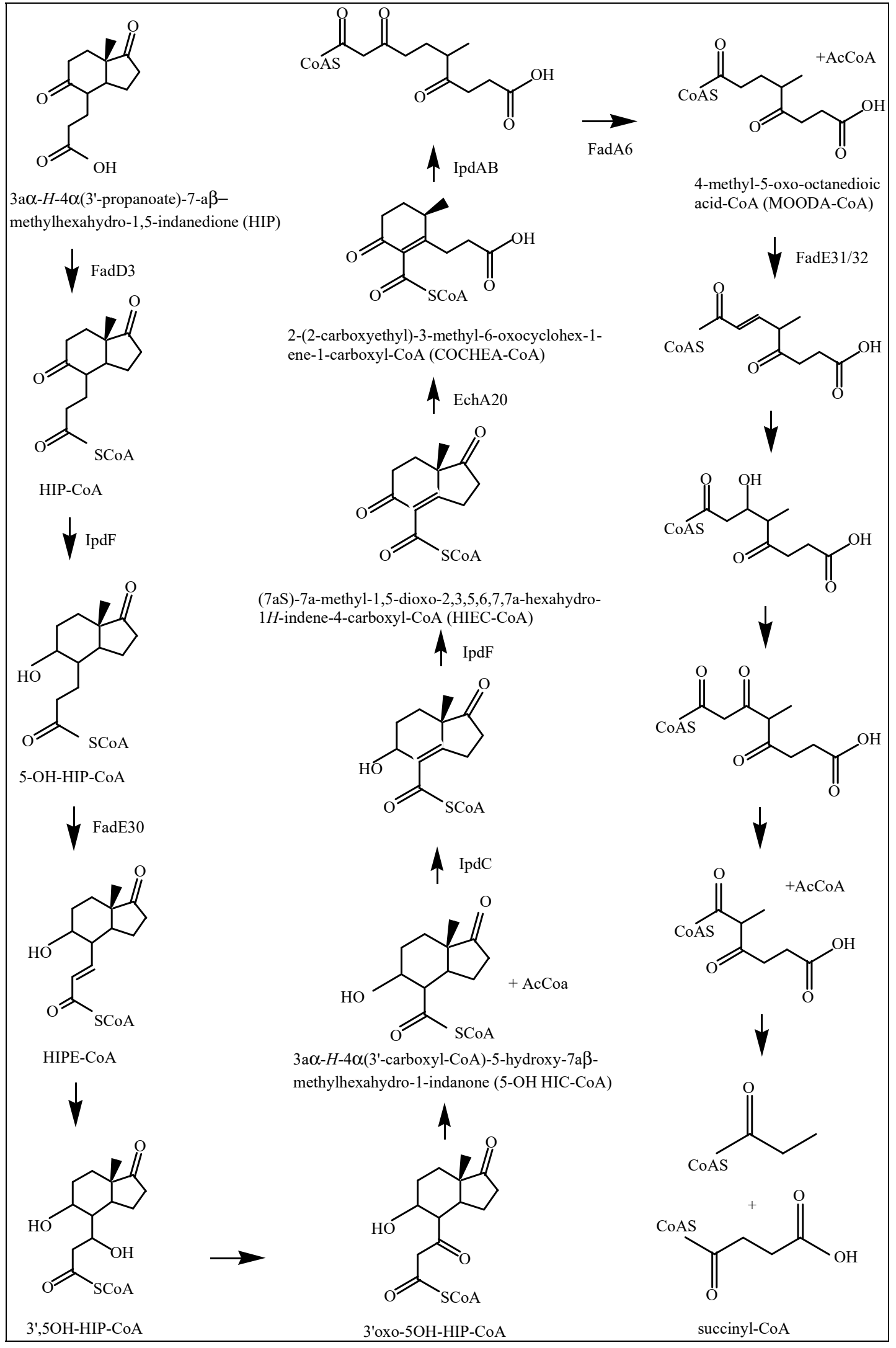

Figure 3. Proposed catabolic pathway of HIP [27]. If known, the enzymes involved in each reaction are depicted by arrows. 


\subsection{Genes/Proteins Involved in Cholesterol Catabolism in M. Tuberculosis H37Rv}

Based on literature, 152 genes/proteins were found to be involved in cholesterol breakdown in M. tuberculosis H37Rv (Table 1). These genes/proteins can be classified into four different categories.

\subsubsection{Genes Predicted to be Specifically Required for Growth on Cholesterol}

Griffin et al. [26] identified 96 genes that are important for the growth of M. tuberculosis on cholesterol through a deep sequencing-based mapping approach (Table 1). Independent studies confirm the genes identified to be important for $M$. tuberculosis growth on cholesterol $[19,22,25,29,30,41]$. A standalone set of genes/proteins predicted to be specifically required for growth on cholesterol is presented in Table S1.

2.2.2. Cholesterol Catabolic Genes Proven to be or Predicted to be Essential for Survival of M. Tuberculosis in Macrophage Cells and in Murine Infection

In the article by Ouellet et al. [19], some of the cholesterol catabolic genes of M. tuberculosis were specified as genes proven to be essential for survival in macrophage cells and in murine infection (Table 1), or genes predicted to be essential for survival in macrophage cells and in murine infection (Table 1). Of the 24 genes listed in Table 1 that are proven to be essential for survival in macrophage cells and in murine infection, 17 genes were predicted to be specifically required for growth on cholesterol by Griffin et al. [26] and other studies [22,25,26,29,30,42]. A standalone set of genes/proteins proven to be essential for survival of $M$. tuberculosis in macrophage cells and in murine infection are presented in Table S2. Genes predicted to be essential for survival of $M$. tuberculosis in macrophage cells and in murine infection are presented in Table S3.

\subsubsection{Genes/Proteins that are Up-Regulated during Growth on Cholesterol}

Van Der Geize et al. [25] predicted a total of 28 genes to be involved in cholesterol catabolism in M. tuberculosis H37Rv. Fifty-one genes specifically expressed during growth on cholesterol in Rhodococcus jostii are also found in an 82-gene cluster in the M. tuberculosis and M. bovis bacillus Calmette-Guérin (BCG) genomes. To annotate the cholesterol catabolic genes, the researchers compared the sequence similarity of the gene products of $R$. jostii RHA1 and M. tuberculosis H37Rv strains and compiled a table with 28 genes annotated for $M$. tuberculosis H37Rv (Table 1). Independent studies confirmed the importance of these genes in cholesterol catabolism by M. tuberculosis $[19,22,26,30]$. Out of the 28 genes, 18 were predicted to be specifically required for growth on cholesterol; 10 of these genes were proven to be essential for survival of $M$. tuberculosis in macrophage cells and in murine infection and 3 were predicted to be essential for survival of $M$. tuberculosis in macrophage cells and in murine infection (Table 1). A standalone set of genes/proteins predicted to be involved in cholesterol catabolism is presented in Table S4.

2.2.4. Genes Involved in Cholesterol Catabolism by M. Tuberculosis H37Rv, but Not Confirmed or Predicted to Be Essential

Based on literature, 40 genes/proteins were found to be involved in cholesterol catabolism by M. tuberculosis H37Rv, but were not confirmed or predicted to be essential according to the published data $[19,22,25,30,34,41,43]$ (Table 1). A standalone set of genes/proteins involved in cholesterol catabolism in M. tuberculosis H37Rv is presented in Table S5. 
Table 1. List of genes/proteins selected for determining mycobacterial species' ability to utilize cholesterol. A standalone set of genes representing different categories is presented in Tables S1-S5.

\begin{tabular}{|c|c|c|}
\hline Gene Name & Gene Number & Protein Name \\
\hline$m c e 4 E / l p r N$ & $R v 3495 c^{a, c, d}$ & Mce4 transport system \\
\hline$m c e 4 C$ & $R v 3497 c^{a, c, d}$ & Mce4 transport system \\
\hline$m c e 4 A$ & $R v 3499^{a, c, d}$ & Mce4 transport system \\
\hline yrb4A/YrbE4A/supA & $R v 3501 c^{a, c, d}$ & possible $\mathrm{ABC}$ transporter (Sterol uptake permease subunit) \\
\hline$h s d 4 A$ & $R v 3502 c^{a, c, d}$ & $17 \beta$-hydroxysteroid dehydrogenase (17 $\beta$-HSD) \\
\hline$k \operatorname{sh} A$ & $R v 3526^{a, c, d}$ & kerosteroid- $9 \alpha$-hydroxylase, oxygenase \\
\hline$h s a F$ & $R v 3534 c^{a, c, d}$ & probable 4-hydroxy-2-oxovalerate aldolase / 4-hydroxy-2-ketovalerate aldolase \\
\hline$k s t D$ & $R v 3537^{b, c, d}$ & 3-ketosteroid- $\Delta 1$-dehydrogenase ( $\Delta 1$-KSTD) \\
\hline fadE28 & $R v 3544 c c^{a, b, c}$ & probable acyl-CoA dehydrogenase \\
\hline$i p d A$ & $R v 3551^{a, b, c}$ & ATP-dependent CoA transferase $\alpha$ subunit \\
\hline fadE30 & $R v 3560 c c^{a, b, c}$ & probable acyl-CoA dehydrogenase \\
\hline fadE32 & $R v 3563^{a, b, c}$ & probable acyl-CoA dehydrogenase \\
\hline$h s a C$ & $R v 3568 c^{a, c, d}$ & 3,4-DHSA dioxygenase \\
\hline$h s a D$ & $R v 3569 c c^{b, c, d}$ & 4,9-DHSA hydrolase \\
\hline$h s a A$ & $R v 3570 c^{b, c, d}$ & 3-hydroxy-9,10-seconandrost-1,3,5(10)-triene-9,17-dione hydroxylase (3-HSA hydroxylase, reductase) \\
\hline$k \operatorname{sh} B$ & $R v 3571^{a, c, d}$ & ketosteroid- $9 \alpha$-hydroxylase, reductase \\
\hline$m c e 4 F$ & $R v 3494 c^{c, d}$ & Mce4 transport system \\
\hline$m c e 4 D$ & $R v 3496 c^{c, d}$ & Mce4 transport system \\
\hline$m c e 4 B$ & $R v 3498 c^{c, d}$ & Mce4 transport system \\
\hline yrb4B/YrbE4B/supB & $R v 3500 c^{c, d}$ & possible $\mathrm{ABC}$ transporter (Sterol uptake permease subunit) \\
\hline fadD19 & $R v 3515 c^{c, d}$ & probable fatty-acid-CoA ligase \\
\hline ltp3 & $R v 3523^{a, d}$ & probable ketoacyl-CoA thiolase \\
\hline$h s a E$ & $R v 3536 c^{c, d}$ & probable hydratase / 2-hydroxypentadienoate hydratase \\
\hline \multirow[t]{2}{*}{ ltp2 } & $R v 3540 c^{a, c}$ & probable ketoacyl-CoA thiolase \\
\hline & $R v 3542 c^{a, c}$ & CHP / putative enoyl-CoA hydratase \\
\hline cyp125 & $R v 3545 c^{a, c}$ & cytochrome P450 \\
\hline fadA5 & $R v 3546^{a, c}$ & acetoacetyl-CoA thiolase \\
\hline fadA6 & $R v 3556 c^{a, b}$ & acetoacetyl-CoA thiolase \\
\hline ppiA & $R v 0009^{c}$ & iron-regulated peptidyl-prolyl cis-trans isomerase A \\
\hline fadD10 & $R v 0099^{e}$ & fatty acid-CoA synthase \\
\hline$p t b B$ & $R v 0153 c^{c}$ & phosphotyrosine protein phosphatase PTPB (protein-tyrosine-phosphatase) (PTPase) \\
\hline mmpL11 & $R v 0202 c^{c}$ & transmembrane transport protein MmpL11 \\
\hline fadE5 & $R v 0244 c^{c}$ & acyl-CoA dehydrogenase \\
\hline$m g t E$ & $R v 0362^{c}$ & $\mathrm{Mg} 2+$ transport transmembrane protein $\mathrm{MgtE}$ \\
\hline metZ & $\operatorname{Rv0391^{c}}$ & O-succinylhomoserine sulfhydrylase \\
\hline mmpL4 & $R v 0450 c^{c}$ & transmembrane transport protein $\mathrm{MmpL4}$ \\
\hline \multirow[t]{3}{*}{$f a d B 2$} & $R v 0468^{e}$ & hydroxybutyryl-CoA dehydrogenase \\
\hline & $R v 0485^{c}$ & transcriptional regulatory protein \\
\hline & $R v 0495 c^{c}$ & $\mathrm{HP}$ \\
\hline$m k l$ & $R v 0655^{c}$ & ribonucleotide $\mathrm{ABC}$ transporter $\mathrm{ATP}$-binding protein \\
\hline$p q q E$ & $\operatorname{Rv0693^{c}}$ & coenzyme PQQ synthesis protein E \\
\hline \multirow[t]{3}{*}{ lldD1 } & $R v 0694^{c}$ & L-lactate dehydrogenase (cytochrome) LldD1 \\
\hline & $\operatorname{Rv0695^{c}}$ & $\mathrm{HP}$ \\
\hline & $R v 0696^{c}$ & membrane sugar transferase \\
\hline \multirow[t]{3}{*}{$a d h B$} & $R v 0761 c^{c}$ & zinc-containing alcohol dehydrogenase NAD dependent ADHB \\
\hline & $R v 0805^{c}$ & HP \\
\hline & $R v 0876 c^{c}$ & transmembrane protein \\
\hline \multirow[t]{6}{*}{ echA9 } & $R v 1071 c^{c}$ & 3-hydroxyisobutyryl-CoA hydrolase \\
\hline & $R v 1084^{c}$ & $\mathrm{HP}$ \\
\hline & $R v 1096^{c}$ & glycosyl hydrolase \\
\hline & $R v 1106 c^{e}$ & $3 \beta-\mathrm{HSD}$ \\
\hline & $R v 1129 c^{c}$ & transcriptional regulator protein \\
\hline & $R v 1130^{c}$ & $\mathrm{HP}$ \\
\hline gltA1 & $\operatorname{Rv} 1131^{c}$ & citrate synthase \\
\hline
\end{tabular}


Table 1. Cont.

\begin{tabular}{|c|c|c|}
\hline Gene Name & Gene Number & Protein Name \\
\hline mmpL10 & $R v 1183^{c}$ & transmembrane transport protein MmpL10 \\
\hline fadD36 & $R v 1193^{c}$ & acyl-CoA synthetase \\
\hline \multirow[t]{3}{*}{$m b t N($ fadE14) } & $R v 1346^{e}$ & acyl-CoA dehydrogenase \\
\hline & $R v 1428 c^{c}$ & $\mathrm{HP}$ \\
\hline & $R v 1432^{c}$ & dehydrogenase \\
\hline \multirow[t]{3}{*}{$b c p B$} & $R v 1608 c^{c}$ & peroxidoxin $\mathrm{BcpB}$ \\
\hline & $R v 1626^{c}$ & two-component system transcriptional regulator \\
\hline & $R v 1627 c^{c}$ & lipid-transfer protein \\
\hline \multirow[t]{4}{*}{ fadB3 } & $R v 1715^{e}$ & hydroxybutyryl-CoA dehydrogenase \\
\hline & $R v 1798^{c}$ & HP \\
\hline & $R v 1906 c^{c}$ & $\mathrm{HP}$ \\
\hline & $R v 1919 c^{c}$ & HP \\
\hline$m c e 3 R$ & $R v 1963 c^{c}$ & transcriptional repressor (probably TETR-family) MCE3R \\
\hline \multirow[t]{4}{*}{ pks12 } & $R v 2048 c^{c}$ & polyketide synthase pks12 \\
\hline & $R v 2118 c^{c}$ & RNA methyltransferase \\
\hline & $R v 2206^{c}$ & transmembrane protein \\
\hline & $R v 2239^{c}{ }^{c}$ & HP \\
\hline eis & $R v 2416 c^{c}$ & $\mathrm{HP}$ \\
\hline \multirow[t]{2}{*}{ tig } & $R v 2462 c^{c}$ & trigger factor \\
\hline & $R v 2506^{c}$ & TetR family transcriptional regulator \\
\hline \multirow[t]{3}{*}{ fadD9 } & $R v 2590^{e}$ & fatty acid-CoA synthase \\
\hline & $R v 2668^{c}$ & HP \\
\hline & $R v 2681^{c}$ & HP \\
\hline $\operatorname{ars} A$ & $R v 2684^{c}$ & arsenic-transport integral membrane protein ArsA \\
\hline \multirow{2}{*}{$\operatorname{sig} B$} & $R v 2710^{c}$ & RNA polymerase sigma factor SigB \\
\hline & $R v 2799^{c}$ & $\mathrm{HP}$ \\
\hline$p k n I$ & $R v 2914 c^{c}$ & transmembrane serine/threonine-protein kinase I \\
\hline \multirow[t]{2}{*}{ mutT1 } & $R v 2985^{c}$ & hydrolase MutT1 \\
\hline & $R v 3050 c^{c}$ & AsnC family transcriptional regulator \\
\hline fadE22 & $R v 3061 c^{e}$ & acyl-CoA dehydrogenase \\
\hline fadE24 & $R v 3139^{e}$ & acyl-CoA dehydrogenase \\
\hline fadE23 & $R v 3140^{e}$ & acyl-CoA dehydrogenase \\
\hline fadE25 & $R v 3274 c^{c}$ & acyl-CoA dehydrogenase FADE25 \\
\hline choD & $R v 3409^{d}$ & cholesterol oxidase \\
\hline \multirow[t]{4}{*}{$g c p$} & $R v 3419^{c}{ }^{c}$ & putative DNA-binding/iron metalloprotein/AP endonuclease \\
\hline & $R v 3421 c^{c}$ & HP \\
\hline & $R v 3492 c^{c}$ & CHP MCE associated protein \\
\hline & $R v 3493 c^{c}$ & CHP MCE associated protein \\
\hline$f d x D$ & $R v 3503 c^{e}$ & probable ferredoxin \\
\hline fadE26 & $R v 3504^{d}$ & probable acyl-CoA dehydrogenase \\
\hline fadE27 & $R v 3505^{d}$ & probable acyl-CoA dehydrogenase \\
\hline fadD17 & $R v 3506^{d}$ & possible fatty-acid-CoA ligase \\
\hline PE PGRS53 & $R v 3507^{e}$ & PE PGRS family \\
\hline PE PGRS54 & $R v 3508^{e}$ & PE PGRS family \\
\hline \multirow[t]{2}{*}{$i l v X$} & $R v 3509 c^{e}$ & probable acetohydroxy-acid synthase \\
\hline & $R v 3510 c^{e}$ & $\mathrm{CHP}$ \\
\hline PE PGRS55 & $R v 3511^{e}$ & PE PGRS family \\
\hline PE PGRS56 & $R v 3512^{e}$ & PE PGRS family \\
\hline fadD18 & $R v 3513 c^{e}$ & possible fatty-acid-CoA ligase \\
\hline PE PGRS57 & $\operatorname{Rv} 3514^{e}$ & PE PGRS family \\
\hline echA19 & $R v 3516^{d}$ & possible enoyl-CoA hydratase \\
\hline whiB3 & $R v 3517^{e}$ & conserved hypothetical protein (CHP) / transcription factor \\
\hline \multirow[t]{4}{*}{ cyp142 } & $R v 3518 c^{e}$ & cytochrome P450 \\
\hline & $R v 3519^{a}$ & $\mathrm{CHP}$ \\
\hline & $R v 3520 c^{e}$ & coenzyme F420-dependent oxidoreductase \\
\hline & $R v 3521^{e}$ & $\mathrm{CHP}$ \\
\hline
\end{tabular}


Table 1. Cont.

\begin{tabular}{|c|c|c|}
\hline Gene Name & Gene Number & Protein Name \\
\hline \multirow[t]{8}{*}{ ltp4 } & $R v 3522^{d}$ & probable ketoacyl-CoA thiolase \\
\hline & $R v 3524^{e}$ & probable conserved membrane protein \\
\hline & $R v 3525 c^{e}$ & possible siderophore binding protein \\
\hline & $R v 3527^{a}$ & hypothetical protein (HP) \\
\hline & $R v 3528 c^{e}$ & $\mathrm{HP}$ \\
\hline & $R v 3529 c^{e}$ & $\mathrm{CHP}$ \\
\hline & $R v 3530 c^{e}$ & possible oxidoreductase \\
\hline & $R v 3531 c^{c}$ & hypothetical protein \\
\hline PPE61 & $\operatorname{Rv3532^{e}}$ & PPE family \\
\hline PPE62 & $R v 3533 c^{e}$ & PPE family \\
\hline$h s a G$ & $R v 3535 c^{d}$ & probable aldehyde dehydrogenase \\
\hline$h s d 4 B$ & $R v 3538^{d}$ & probable enoyl-CoA hydratase \\
\hline \multirow[t]{2}{*}{ PPE63 } & Rv3539e & PE \\
\hline & $R v 3541 c^{a}$ & CHP / putative enoyl-CoA hydratase \\
\hline \multirow[t]{4}{*}{ fadE29 } & $R v 3543 c^{c}$ & probable acyl-CoA dehydrogenase \\
\hline & $\operatorname{Rv} 3547^{e}$ & $\mathrm{CHP}$ \\
\hline & $R v 3548 c^{c}$ & probable short chain dehydrogenase/reductase \\
\hline & $R v 3549 c^{c}$ & probable short chain dehydrogenase/reductase \\
\hline echA20 & $\operatorname{Rv} 3550^{e}$ & possible enoyl-CoA hydratase \\
\hline \multirow[t]{2}{*}{$i p d B$} & $R v 3552^{a}$ & ATP-dependent CoA transferase $\beta$ subunit \\
\hline & $\operatorname{Rv} 3553^{c}$ & possible oxidoreductase / 2-nitropropane dioxygenase \\
\hline \multirow[t]{2}{*}{$f d x B$} & $\operatorname{Rv} 3554^{e}$ & possible electron transfer protein / ferredoxin \\
\hline & $R v 3555 c^{e}$ & $\mathrm{CHP}$ \\
\hline$k s t R 2$ & $R v 3557 c^{e}$ & Tet-R transcriptional regulator (repressor) \\
\hline \multirow[t]{2}{*}{ PPE64 } & Rv3558e & PPE \\
\hline & $R v 3559^{c}{ }^{c}$ & probable oxidoreductase \\
\hline fadD3 & $\operatorname{Rv} 3561^{c}$ & acyl-CoA synthetase (AMP forming) \\
\hline fadE31 & $R v 3562^{e}$ & probable acyl-CoA dehydrogenase \\
\hline fadE33 & $R v 3564^{c}$ & probable acyl-CoA dehydrogenase \\
\hline \multirow[t]{2}{*}{ aspB } & $\operatorname{Rv3565^{e}}$ & possible aspartate aminotransferase \\
\hline & $R v 3566 A^{e}$ & $\mathrm{CHP}$ \\
\hline nhoA/nat & $R v 3566 c^{e}$ & arylamine $\mathrm{N}$-acetyltransferase \\
\hline \multirow[t]{2}{*}{$h s a B$} & $R v 3567 c^{d}$ & 3-hydroxy-9,10-seconandrost-1,3,5(10)-triene-9,17-dione hydroxylase (3-HSA hydroxylase, reductase) \\
\hline & $\operatorname{Rv3572^{c}}$ & HP \\
\hline fadE34 & $R v 3573 c^{c}$ & probable acyl-CoA dehydrogenase \\
\hline \multirow[t]{3}{*}{$k s t R$} & $\operatorname{Rv3574^{a}}$ & Tet-R transcriptional regulator (repressor) \\
\hline & $R v 3575 c^{c}$ & transcriptional regulatory protein LacI-family \\
\hline & $\operatorname{Rv3779^{c}}$ & transmembrane protein alanine and leucine rich \\
\hline papA2 & $R v 3820 c^{c}$ & polyketide synthase associated protein PapA2 \\
\hline papA1 & $R v 3824 c^{c}$ & polyketide synthase associated protein \\
\hline pks2 & $R v 3825 c^{c}$ & polyketide synthase PKS2 \\
\hline $\operatorname{sig} M$ & $R v 3911^{c}$ & RNA polymerase sigma factor SigM \\
\hline
\end{tabular}

Notes: ${ }^{a}$ Genes proven to be essential for survival in macrophage cells and in murine infection. ${ }^{b}$ Genes predicted to be essential for survival in macrophage cells and in murine infection. ${ }^{c}$ Genes predicted to be specifically required for growth on cholesterol. ${ }^{d}$ Genes predicted to be involved in cholesterol catabolism compiled from annotation of RHA1, H37Rv and BCG (bacillus Calmette-Guérin) genes assigned to cholesterol pathway. ${ }^{e}$ Genes involved in cholesterol catabolism by $M$. tuberculosis $\mathrm{H} 37 \mathrm{Rv}$ but not confirmed or predicted as essential, according to the published data. Abbreviations: 3-HSA = 3-hydroxy-9,10-secoandrosta-1,3,5(10)-triene-9,17-dione; 3,4-DHSA =

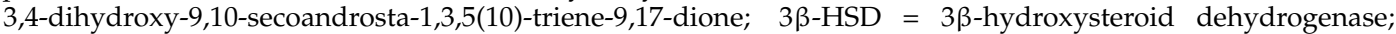
4,9-DHSA hydrolase = 4,5-9,10-diseco-3-hydroxy-5,9,17-trioxoandrosta-1(10),2-dien-4-oic acid; 17 $\beta$-HSD = 17 $\beta$ hydroxysteroid dehydrogenase; $\triangle 1$-KSTD $=3$-ketosteroid- $\Delta 1$-dehydrogenase; $\mathrm{ABC}=\mathrm{ATP}$-binding cassette; $\mathrm{ADH}=$ alcohol dehydrogenase; $\mathrm{AMP}=$ adenosine monophosphate; $\mathrm{AP}=$ apurinic/apyrimidinic; $\mathrm{ATP}=$ adenosine triphosphate; $\mathrm{Bcp}=$ bacterioferritin comigratory protein; $\mathrm{CHP}=$ conserved hypothetical protein; $\mathrm{CoA}=$ coenzyme A; DNA = deoxyribonucleic acid; $\mathrm{HP}=$ hypothetical protein; $\mathrm{LldD}=$ L-lactate dehydrogenase; $\mathrm{MCE}=$ mammalian cell entry; $\mathrm{MgtE}=\mathrm{Mg} 2+$ transport transmembrane protein; $\mathrm{MmpL}=$ Mycobacterium membrane protein laboratory; $\mathrm{NAD}=$ nicotinamide adenine dinucleotide; $\mathrm{PE}=$ protein family with highly conserved Proline-Glutamate residues near the start of their encoded proteins; PGRS = polymorphic GC-rich-repetitive sequence; pks = polyketide synthase; $\mathrm{PPE}=$ protein family with highly conserved proline-proline-glutamate; $\mathrm{PQQ}=$ pyrrolo-quinoline quinone; $\mathrm{PTP} / \mathrm{PTPase}=$ phosphotyrosine protein phosphatase $/$ protein-tyrosine-phosphatase; $\mathrm{RNA}=$ ribonucleic acid; TetR/TETR = tetracycline repressor. 


\subsection{Key Cholesterol Catabolic Genes/Proteins are Not Found in a Large Number of Mycobacterial Species}

Because of the omission of 1 gene (Rv3512, as mentioned in Section 3.3.4), 151 genes/proteins were selected to assess the different mycobacterial species' ability for cholesterol catabolism instead of the initial 152 (Table 1). Mycobacterial species' ability to catabolize cholesterol was predicted based on the presence of two categories of genes/proteins (i.e., cholesterol catabolic genes/proteins proven or predicted to be essential or specifically required for growth of $M$. tuberculosis $\mathrm{H} 37 \mathrm{Rv}$ on cholesterol). Comprehensive comparative analysis of different categories of genes/proteins in mycobacterial species is presented in Table 2 .

Table 2. Comparative analysis of cholesterol degrading genes/proteins in mycobacterial species. M. tuberculosis H37Rv homologs belonging to different categories not found in mycobacterial species were listed under different categories. The relevant data on BLAST analysis, homolog proteins and protein family analysis are presented in Supplementary Datasets 1-3, respectively. The cholesterol catabolic ability of mycobacterial species was predicted following the presence of genes/proteins that are proven to be essential, and predicted to be essential or specifically required for M. tuberculosis H37Rv growth on cholesterol.

\begin{tabular}{|c|c|c|c|c|c|}
\hline \multirow[b]{2}{*}{$\begin{array}{l}\text { Organism } \\
\text { Code }\end{array}$} & \multicolumn{4}{|c|}{ H37Rv Homolog(s) Not Found Relating to Cholesterol Catabolism } & \multirow{2}{*}{$\begin{array}{l}\text { Ability to } \\
\text { Degrade } \\
\text { Cholesterol }\end{array}$} \\
\hline & $\begin{array}{l}\text { Proven to Be } \\
\text { Essential }\end{array}$ & $\begin{array}{l}\text { Predicted to Be Essential or } \\
\text { Specifically Required }\end{array}$ & $\begin{array}{l}\text { Predicted to Be } \\
\text { Involved }\end{array}$ & $\begin{array}{l}\text { Involved but Not Proven or } \\
\text { Predicted to Be Essential }\end{array}$ & \\
\hline \multicolumn{6}{|c|}{ Mycobacterium tuberculosis complex (MTBC) } \\
\hline mtu & None & None & None & None & Positive \\
\hline mtv & None & None & None & None & Positive \\
\hline mtc & None & None & None & Rv3555c & Positive \\
\hline mra & None & None & None & None & Positive \\
\hline $\mathrm{mtf}$ & None & None & None & Rv3566A & Positive \\
\hline $\mathrm{mtb}$ & None & None & None & Rv3566A & Positive \\
\hline mtk & None & None & None & Rv3566A & Positive \\
\hline $\mathrm{mtz}$ & None & None & None & Rv3566A & Positive \\
\hline $\mathrm{mtg}$ & None & $\begin{array}{l}\text { Rv1084 } \\
\text { Rv2799 }\end{array}$ & None & None & No prediction \\
\hline mti & Rv3526 & $\begin{array}{c}\text { Rv0153c } \\
\text { Rv0485 } \\
\text { Rv0805 } \\
\text { Rv0876c } \\
\text { Rv2416c } \\
\text { Rv2681 } \\
\text { Rv3526 } \\
\text { Rv3531c }\end{array}$ & Rv3526 & None & No prediction \\
\hline mte & None & $\begin{array}{c}\text { Rv0805 } \\
\text { Rv1919c } \\
\end{array}$ & None & Rv3566A & No prediction \\
\hline mtur & None & None & None & None & Positive \\
\hline $\mathrm{mtl}$ & None & $\begin{array}{l}\text { Rv0805 } \\
\text { Rv1919c }\end{array}$ & None & Rv3566A & No prediction \\
\hline mto & None & None & None & None & Positive \\
\hline mtd & None & None & None & None & Positive \\
\hline $\mathrm{mtn}$ & None & Rv0805 & None & Rv3566A & No prediction \\
\hline $\mathrm{mtj}$ & None & None & None & Rv3566A & Positive \\
\hline mtub & None & None & None & None & Positive \\
\hline mtuc & None & $\begin{array}{c}\text { Rv0485 } \\
\text { Rv0695 } \\
\text { Rv1084 } \\
\text { Rv1130 } \\
\text { Rv1432 } \\
\text { Rv2416c } \\
\text { Rv2681 } \\
\text { Rv3536c } \\
\text { Rv3779 }\end{array}$ & Rv3536c & $\begin{array}{c}\text { Rv3521 } \\
\text { Rv3566A }\end{array}$ & No prediction \\
\hline mtue & None & Rv2681 & None & Rv3566A & No prediction \\
\hline $\mathrm{mtx}$ & None & None & None & None & Positive \\
\hline
\end{tabular}


Table 2. Cont

\begin{tabular}{|c|c|c|c|c|c|}
\hline \multirow[b]{2}{*}{$\begin{array}{l}\text { Organism } \\
\text { Code }\end{array}$} & \multicolumn{4}{|c|}{ H37Rv Homolog(s) Not Found Relating to Cholesterol Catabolism } & \multirow{2}{*}{$\begin{array}{l}\text { Ability to } \\
\text { Degrade } \\
\text { Cholesterol }\end{array}$} \\
\hline & $\begin{array}{l}\text { Proven to Be } \\
\text { Essential }\end{array}$ & $\begin{array}{l}\text { Predicted to Be Essential or } \\
\text { Specifically Required }\end{array}$ & $\begin{array}{l}\text { Predicted to Be } \\
\text { Involved }\end{array}$ & $\begin{array}{l}\text { Involved but Not Proven or } \\
\text { Predicted to Be Essential }\end{array}$ & \\
\hline \multicolumn{6}{|c|}{ Mycobacterium tuberculosis complex (МТВC) } \\
\hline mtuh & None & $\begin{array}{c}\text { Rv0485 } \\
\text { Rv0876c } \\
\text { Rv1084 } \\
\text { Rv1096 } \\
\text { Rv1129c } \\
\text { Rv2416c } \\
\text { Rv3531c }\end{array}$ & None & None & No prediction \\
\hline mtul & None & None & None & Rv3566A & Positive \\
\hline mtut & None & None & None & None & Positive \\
\hline mtuu & None & None & None & None & Positive \\
\hline $\mathrm{mtq}$ & None & None & None & None & Positive \\
\hline mbo & None & None & None & None & Positive \\
\hline $\mathrm{mbb}$ & None & None & None & None & Positive \\
\hline $\mathrm{mbt}$ & None & None & None & None & Positive \\
\hline $\mathrm{mbm}$ & None & None & None & None & Positive \\
\hline mbk & None & None & None & Rv3566A & Positive \\
\hline $\operatorname{mbx}$ & None & $\begin{array}{l}\text { Rv0805 } \\
\text { Rv2206 } \\
\end{array}$ & None & $\begin{array}{l}\text { Rv3566A } \\
\text { Rv3566c }\end{array}$ & No prediction \\
\hline $\mathrm{mbz}$ & None & None & None & None & Positive \\
\hline maf & None & None & None & Rv3528c & Positive \\
\hline mce & None & Rv1130 & None & None & No prediction \\
\hline mcq & None & None & None & None & Positive \\
\hline $\mathrm{mcv}$ & None & None & None & None & Positive \\
\hline $\mathrm{mcx}$ & None & None & & Rv3566A & Positive \\
\hline $\mathrm{mcz}$ & None & None & None & $\begin{array}{c}\text { Rv3517 } \\
\text { Rv3528c } \\
\text { Rv3566A }\end{array}$ & Positive \\
\hline \multicolumn{6}{|c|}{ Mycobacterium chelonae-abscessus complex (MCAC) } \\
\hline mab & Rv3519 & $\begin{array}{l}\text { Rv0876c } \\
\text { Rv1906c } \\
\text { Rv2684 }\end{array}$ & None & $\begin{array}{c}\text { Rv3507 } \\
\text { Rv3508 } \\
\text { Rv3511 } \\
\text { Rv3514 } \\
\text { Rv3524 } \\
\text { Rv3528c } \\
\text { Rv3566A }\end{array}$ & No prediction \\
\hline mabb & Rv3519 & $\begin{array}{c}\text { Rv0876c } \\
\text { Rv1906c } \\
\text { Rv2684 } \\
\text { Rv3575c }\end{array}$ & None & $\begin{array}{c}\text { Rv3507 } \\
\text { Rv3508 } \\
\text { Rv3511 } \\
\text { Rv3514 } \\
\text { Rv3524 } \\
\text { Rv3528c } \\
\text { Rv3566A }\end{array}$ & No prediction \\
\hline $\mathrm{mmv}$ & Rv3519 & $\begin{array}{l}\text { Rv0876c } \\
\text { Rv2684 } \\
\text { Rv3575c }\end{array}$ & None & $\begin{array}{c}\text { Rv3507 } \\
\text { Rv3508 } \\
\text { Rv3511 } \\
\text { Rv3514 } \\
\text { Rv3524 } \\
\text { Rv3528c } \\
\text { Rv3566A } \\
\end{array}$ & No prediction \\
\hline may & Rv3519 & $\begin{array}{c}\text { Rv1906c } \\
\text { Rv2684 }\end{array}$ & None & $\begin{array}{c}\text { Rv3507 } \\
\text { Rv3508 } \\
\text { Rv3511 } \\
\text { Rv3514 } \\
\text { Rv3524 } \\
\text { Rv3528c } \\
\text { Rv3566A }\end{array}$ & No prediction \\
\hline mabo & Rv3519 & $\begin{array}{l}\text { Rv1906c } \\
\text { Rv2684 }\end{array}$ & None & $\begin{array}{c}\text { Rv3507 } \\
\text { Rv3508 } \\
\text { Rv3511 } \\
\text { Rv3514 } \\
\text { Rv3524 } \\
\text { Rv3528c } \\
\text { Rv3566A }\end{array}$ & No prediction \\
\hline
\end{tabular}


Table 2. Cont.

\begin{tabular}{|c|c|c|c|c|c|}
\hline \multirow[b]{2}{*}{$\begin{array}{l}\text { Organism } \\
\text { Code }\end{array}$} & \multicolumn{4}{|c|}{ H37Rv Homolog(s) Not Found Relating to Cholesterol Catabolism } & \multirow{2}{*}{$\begin{array}{l}\text { Ability to } \\
\text { Degrade } \\
\text { Cholesterol }\end{array}$} \\
\hline & $\begin{array}{l}\text { Proven to Be } \\
\text { Essential }\end{array}$ & $\begin{array}{l}\text { Predicted to Be Essential or } \\
\text { Specifically Required }\end{array}$ & $\begin{array}{l}\text { Predicted to Be } \\
\text { Involved }\end{array}$ & $\begin{array}{l}\text { Involved but Not Proven or } \\
\text { Predicted to Be Essential }\end{array}$ & \\
\hline \multicolumn{6}{|c|}{ Mycobacterium chelonae-abscessus complex (MCAC) } \\
\hline mabl & Rv3519 & $\begin{array}{l}\text { Rv0876c } \\
\text { Rv1906c } \\
\text { Rv2684 } \\
\text { Rv3575c }\end{array}$ & None & $\begin{array}{c}\text { Rv3507 } \\
\text { Rv3508 } \\
\text { Rv3511 } \\
\text { Rv3514 } \\
\text { Rv3517 } \\
\text { Rv3524 } \\
\text { Rv3528c } \\
\text { Rv3566A }\end{array}$ & No prediction \\
\hline maz & Rv3519 & $\begin{array}{l}\text { Rv1906c } \\
\text { Rv2684 }\end{array}$ & None & $\begin{array}{c}\text { Rv3507 } \\
\text { Rv3508 } \\
\text { Rv3511 } \\
\text { Rv3514 } \\
\text { Rv3524 } \\
\text { Rv3528c } \\
\text { Rv3566A }\end{array}$ & No prediction \\
\hline mak & Rv3519 & $\begin{array}{l}\text { Rv1906c } \\
\text { Rv2684 } \\
\text { Rv3575c }\end{array}$ & None & $\begin{array}{c}\text { Rv3507 } \\
\text { Rv3508 } \\
\text { Rv3511 } \\
\text { Rv3514 } \\
\text { Rv3524 } \\
\text { Rv3528c } \\
\text { Rv3566A }\end{array}$ & No prediction \\
\hline mys & Rv3519 & $\begin{array}{l}\text { Rv2684 } \\
\text { Rv3575c }\end{array}$ & None & $\begin{array}{c}\text { Rv3507 } \\
\text { Rv3508 } \\
\text { Rv3511 } \\
\text { Rv3514 } \\
\text { Rv3524 } \\
\text { Rv3528c } \\
\text { Rv3566A }\end{array}$ & No prediction \\
\hline myc & Rv3519 & $\begin{array}{l}\text { Rv2684 } \\
\text { Rv3575c }\end{array}$ & None & $\begin{array}{c}\text { Rv3507 } \\
\text { Rv3508 } \\
\text { Rv3511 } \\
\text { Rv3514 } \\
\text { Rv3524 } \\
\text { Rv3528c } \\
\text { Rv3566A } \\
\end{array}$ & No prediction \\
\hline \multicolumn{6}{|c|}{ Mycobacterium avium complex (MAC) } \\
\hline mpa & None & None & None & $\begin{array}{l}\text { Rv3528c } \\
\text { Rv3566A }\end{array}$ & Positive \\
\hline mao & None & Rv0153c & None & $\begin{array}{l}\text { Rv3528c } \\
\text { Rv3566A }\end{array}$ & No prediction \\
\hline mavi & None & $\begin{array}{l}\text { Rv0153c } \\
\text { Rv1084 }\end{array}$ & None & $\begin{array}{l}\text { Rv3528c } \\
\text { Rv3566A }\end{array}$ & No prediction \\
\hline mavu & None & None & None & $\begin{array}{r}\text { Rv3528c } \\
\text { Rv3566A }\end{array}$ & Positive \\
\hline mav & None & Rv3779 & None & $\begin{array}{l}\text { Rv3528c } \\
\text { Rv3566A } \\
\end{array}$ & No prediction \\
\hline mavd & None & Rv0153c & None & $\begin{array}{l}\text { Rv3528c } \\
\text { Rv3566A }\end{array}$ & No prediction \\
\hline mavr & None & None & None & $\begin{array}{r}\text { Rv3528c } \\
\text { Rv3566A } \\
\end{array}$ & Positive \\
\hline mava & None & None & None & $\begin{array}{r}\text { Rv3528c } \\
\text { Rv3566A }\end{array}$ & Positive \\
\hline mit & Rv3519 & None & None & $\begin{array}{l}\text { Rv3528c } \\
\text { Rv3566A }\end{array}$ & No prediction \\
\hline $\operatorname{mir}$ & None & None & None & $\begin{array}{l}\text { Rv3528c } \\
\text { Rv3566A }\end{array}$ & Positive \\
\hline mia & None & None & None & $\begin{array}{l}\text { Rv3528c } \\
\text { Rv3566A }\end{array}$ & Positive \\
\hline mie & None & None & None & $\begin{array}{l}\text { Rv3528c } \\
\text { Rv3566A }\end{array}$ & Positive \\
\hline mid & None & None & None & $\begin{array}{l}\text { Rv3528c } \\
\text { Rv3566A }\end{array}$ & Positive \\
\hline myo & None & None & None & $\begin{array}{l}\text { Rv3528c } \\
\text { Rv3566A }\end{array}$ & Positive \\
\hline $\mathrm{mmm}$ & None & None & None & $\begin{array}{l}\text { Rv3528c } \\
\text { Rv3566A }\end{array}$ & Positive \\
\hline
\end{tabular}


Table 2. Cont.

\begin{tabular}{|c|c|c|c|c|c|}
\hline \multirow[b]{2}{*}{$\begin{array}{l}\text { Organism } \\
\text { Code }\end{array}$} & \multicolumn{4}{|c|}{ H37Rv Homolog(s) Not Found Relating to Cholesterol Catabolism } & \multirow{2}{*}{$\begin{array}{l}\text { Ability to } \\
\text { Degrade } \\
\text { Cholesterol }\end{array}$} \\
\hline & $\begin{array}{l}\text { Proven to Be } \\
\text { Essential }\end{array}$ & $\begin{array}{l}\text { Predicted to Be Essential or } \\
\text { Specifically Required }\end{array}$ & $\begin{array}{l}\text { Predicted to Be } \\
\text { Involved }\end{array}$ & $\begin{array}{l}\text { Involved but Not Proven or } \\
\text { Predicted to Be Essential }\end{array}$ & \\
\hline \multicolumn{6}{|c|}{ Mycobacteria causing leprosy (MCL) } \\
\hline mle & $\begin{array}{c}\text { Rv3523 } \\
\text { Rv3526 } \\
\text { Rv3540c } \\
\text { Rv3551 } \\
\text { Rv3568c } \\
\text { Rv3571 } \\
\text { Rv3519 } \\
\text { Rv3527 } \\
\text { Rv3552 }\end{array}$ & $\begin{array}{c}\text { Rv0153c } \\
\text { Rv0485 } \\
\text { Rv0693 } \\
\text { Rv0695 } \\
\text { Rv1084 } \\
\text { Rv1129c } \\
\text { Rv1130 } \\
\text { Rv2416c } \\
\text { Rv2668 } \\
\text { Rv2799 } \\
\text { Rv3492c } \\
\text { Rv3493c } \\
\text { Rv3526 } \\
\text { Rv3531c } \\
\text { Rv3536c } \\
\text { Rv3540c } \\
\text { Rv3551 } \\
\text { Rv3553 } \\
\text { Rv3568c } \\
\text { Rv3571 }\end{array}$ & $\begin{array}{c}\text { Rv3523 } \\
\text { Rv3526 } \\
\text { Rv3535c } \\
\text { Rv3536c } \\
\text { Rv3568c } \\
\text { Rv3571 }\end{array}$ & $\begin{array}{c}\text { Rv3503c } \\
\text { Rv3510c } \\
\text { Rv3517 } \\
\text { Rv3521 } \\
\text { Rv3524 } \\
\text { Rv3528c } \\
\text { Rv3529c } \\
\text { Rv3554 } \\
\text { Rv3555c } \\
\text { Rv3566A } \\
\text { Rv3566c }\end{array}$ & Negative \\
\hline $\mathrm{mlb}$ & $\begin{array}{c}\text { Rv3523 } \\
\text { Rv3526 } \\
\text { Rv3540c } \\
\text { Rv3551 } \\
\text { Rv3568c } \\
\text { Rv3571 } \\
\text { Rv3519 } \\
\text { Rv3527 } \\
\text { Rv3552 }\end{array}$ & $\begin{array}{c}\text { Rv0153c } \\
\text { Rv0485 } \\
\text { Rv0693 } \\
\text { Rv0695 } \\
\text { Rv1084 } \\
\text { Rv1129c } \\
\text { Rv1130 } \\
\text { Rv2416c } \\
\text { Rv2668 } \\
\text { Rv2799 } \\
\text { Rv3492c } \\
\text { Rv3493c } \\
\text { Rv3526 } \\
\text { Rv3531c } \\
\operatorname{Rv} 3536 c \\
\text { Rv3540c } \\
\text { Rv3551 } \\
\text { Rv3553 } \\
\operatorname{Rv} 3568 c \\
\text { Rv3571 }\end{array}$ & $\begin{array}{c}\text { Rv3523 } \\
\text { Rv3526 } \\
\text { Rv3535c } \\
\text { Rv3536c } \\
\text { Rv3568c } \\
\text { Rv3571 }\end{array}$ & $\begin{array}{c}\text { Rv3503c } \\
\text { Rv3510c } \\
\text { Rv3517 } \\
\text { Rv3521 } \\
\text { Rv3524 } \\
\text { Rv3528c } \\
\text { Rv3529c } \\
\text { Rv3554 } \\
\text { Rv3555c } \\
\text { Rv3566A } \\
\text { Rv3566c }\end{array}$ & Negative \\
\hline \multicolumn{6}{|c|}{ Non-tuberculosis Mycobacterium (NTM) } \\
\hline mul & None & Rv2416c & None & $\begin{array}{l}\text { Rv3517 } \\
\text { Rv3528c } \\
\text { Rv3566A }\end{array}$ & No prediction \\
\hline mjd & None & Rv3575c & None & $\begin{array}{l}\text { Rv3528c } \\
\text { Rv3566A }\end{array}$ & No prediction \\
\hline mmi & None & None & None & $\begin{array}{l}\text { Rv3528c } \\
\text { Rv3566A }\end{array}$ & Positive \\
\hline mli & None & None & None & $\begin{array}{l}\text { Rv3528c } \\
\text { Rv3566A }\end{array}$ & Positive \\
\hline $\mathrm{mkn}$ & None & None & None & $\begin{array}{l}\text { Rv3528c } \\
\text { Rv3566A }\end{array}$ & Positive \\
\hline $\mathrm{mks}$ & None & Rv2462c & None & $\begin{array}{l}\text { Rv3528c } \\
\text { Rv3566A }\end{array}$ & No prediction \\
\hline mki & None & Rv2462c & None & $\begin{array}{l}\text { Rv3528c } \\
\text { Rv3566A }\end{array}$ & No prediction \\
\hline mhad & Rv3534c & $\begin{array}{l}\text { Rv1130 } \\
\text { Rv3534c }\end{array}$ & Rv3534c & $\begin{array}{l}\text { Rv3528c } \\
\text { Rv3566A }\end{array}$ & No prediction \\
\hline
\end{tabular}


Table 2. Cont.

\begin{tabular}{|c|c|c|c|c|c|}
\hline \multirow[b]{2}{*}{$\begin{array}{l}\text { Organism } \\
\text { Code }\end{array}$} & \multicolumn{4}{|c|}{ H37Rv Homolog(s) Not Found Relating to Cholesterol Catabolism } & \multirow{2}{*}{$\begin{array}{l}\text { Ability to } \\
\text { Degrade } \\
\text { Cholesterol }\end{array}$} \\
\hline & $\begin{array}{l}\text { Proven to Be } \\
\text { Essential }\end{array}$ & $\begin{array}{l}\text { Predicted to Be Essential or } \\
\text { Specifically Required }\end{array}$ & $\begin{array}{l}\text { Predicted to Be } \\
\text { Involved }\end{array}$ & $\begin{array}{l}\text { Involved but Not Proven or } \\
\text { Predicted to Be Essential }\end{array}$ & \\
\hline \multicolumn{6}{|c|}{ Saprophytes (SAP) } \\
\hline $\mathrm{msm}$ & None & $\begin{array}{l}\text { Rv0805 } \\
\text { Rv3572 } \\
\text { Rv3779 }\end{array}$ & None & $\begin{array}{c}\text { Rv3507 } \\
\text { Rv3508 } \\
\text { Rv3511 } \\
\text { Rv3514 } \\
\text { Rv3528c } \\
\text { Rv3566A }\end{array}$ & No prediction \\
\hline msg & None & $\begin{array}{l}\text { Rv0805 } \\
\text { Rv3572 } \\
\text { Rv3779 }\end{array}$ & None & $\begin{array}{c}\text { Rv3507 } \\
\text { Rv3508 } \\
\text { Rv3511 } \\
\text { Rv3514 } \\
\text { Rv3528c } \\
\text { Rv3566A } \\
\end{array}$ & No prediction \\
\hline $\mathrm{msb}$ & None & $\begin{array}{l}\text { Rv0805 } \\
\text { Rv3572 } \\
\text { Rv3779 }\end{array}$ & None & $\begin{array}{l}\text { Rv3507 } \\
\text { Rv3508 } \\
\text { Rv3511 } \\
\text { Rv3514 } \\
\text { Rv3528c } \\
\text { Rv3566A }\end{array}$ & No prediction \\
\hline msn & None & $\begin{array}{l}\text { Rv0805 } \\
\text { Rv3493c } \\
\text { Rv3572 } \\
\text { Rv3779 }\end{array}$ & None & $\begin{array}{c}\text { Rv3507 } \\
\text { Rv3508 } \\
\text { Rv3511 } \\
\text { Rv3514 } \\
\text { Rv3528c } \\
\text { Rv3566A }\end{array}$ & No prediction \\
\hline msh & None & $\begin{array}{l}\text { Rv0805 } \\
\text { Rv3572 } \\
\text { Rv3779 }\end{array}$ & None & $\begin{array}{l}\text { Rv3507 } \\
\text { Rv3508 } \\
\text { Rv3511 } \\
\text { Rv3514 } \\
\text { Rv3528c } \\
\text { Rv3566A }\end{array}$ & No prediction \\
\hline msa & None & Rv1130 & None & $\begin{array}{c}\text { Rv3507 } \\
\text { Rv3508 } \\
\text { Rv3511 } \\
\text { Rv3514 } \\
\text { Rv3517 } \\
\text { Rv3528c }\end{array}$ & No prediction \\
\hline mva & None & $\begin{array}{l}\text { Rv0805 } \\
\text { Rv1130 } \\
\text { Rv3572 } \\
\text { Rv3779 }\end{array}$ & None & $\begin{array}{c}\text { Rv3507 } \\
\text { Rv3508 } \\
\text { Rv3511 } \\
\text { Rv3514 } \\
\text { Rv3517 } \\
\text { Rv3528c } \\
\text { Rv3566A }\end{array}$ & No prediction \\
\hline mgi & None & $\begin{array}{l}\text { Rv0805 } \\
\text { Rv1130 } \\
\text { Rv3572 } \\
\text { Rv3779 }\end{array}$ & None & $\begin{array}{c}\text { Rv3507 } \\
\text { Rv3508 } \\
\text { Rv3514 } \\
\text { Rv3528c } \\
\text { Rv3566A }\end{array}$ & No prediction \\
\hline $\mathrm{msp}$ & None & $\begin{array}{c}\text { Rv0805 } \\
\text { Rv1084 } \\
\text { Rv1130 } \\
\text { Rv1919c } \\
\text { Rv3492c } \\
\text { Rv3572 } \\
\text { Rv3779 }\end{array}$ & None & $\begin{array}{l}\text { Rv3507 } \\
\text { Rv3508 } \\
\text { Rv3511 } \\
\text { Rv3514 } \\
\text { Rv3528c } \\
\text { Rv3566A }\end{array}$ & No prediction \\
\hline $\mathrm{mmc}$ & None & $\begin{array}{l}\text { Rv0805 } \\
\text { Rv1130 } \\
\text { Rv3572 } \\
\text { Rv3779 }\end{array}$ & None & $\begin{array}{c}\text { Rv3507 } \\
\text { Rv3508 } \\
\text { Rv3511 } \\
\text { Rv3514 } \\
\text { Rv3528c } \\
\text { Rv3566A }\end{array}$ & No prediction \\
\hline $\mathrm{mkm}$ & None & $\begin{array}{l}\text { Rv0805 } \\
\text { Rv1130 } \\
\text { Rv3572 } \\
\text { Rv3779 }\end{array}$ & None & $\begin{array}{c}\text { Rv3507 } \\
\text { Rv3508 } \\
\text { Rv3511 } \\
\text { Rv3514 } \\
\text { Rv3528c } \\
\text { Rv3566A }\end{array}$ & No prediction \\
\hline
\end{tabular}


Table 2. Cont.

\begin{tabular}{|c|c|c|c|c|c|}
\hline \multirow[b]{2}{*}{$\begin{array}{l}\text { Organism } \\
\text { Code }\end{array}$} & \multicolumn{4}{|c|}{ H37Rv Homolog(s) Not Found Relating to Cholesterol Catabolism } & \multirow{2}{*}{$\begin{array}{l}\text { Ability to } \\
\text { Degrade } \\
\text { Cholesterol }\end{array}$} \\
\hline & $\begin{array}{l}\text { Proven to Be } \\
\text { Essential }\end{array}$ & $\begin{array}{l}\text { Predicted to Be Essential or } \\
\text { Specifically Required }\end{array}$ & $\begin{array}{l}\text { Predicted to Be } \\
\text { Involved }\end{array}$ & $\begin{array}{l}\text { Involved but Not Proven or } \\
\text { Predicted to Be Essential }\end{array}$ & \\
\hline \multicolumn{6}{|c|}{ Saprophytes (SAP) } \\
\hline mjl & None & $\begin{array}{l}\text { Rv0805 } \\
\text { Rv1130 } \\
\text { Rv3572 } \\
\text { Rv3779 }\end{array}$ & None & $\begin{array}{c}\text { Rv3507 } \\
\text { Rv3508 } \\
\text { Rv3511 } \\
\text { Rv3514 } \\
\text { Rv3528c } \\
\text { Rv3566A }\end{array}$ & No prediction \\
\hline $\mathrm{mrh}$ & None & $\begin{array}{l}\text { Rv0805 } \\
\text { Rv1130 } \\
\text { Rv3572 } \\
\text { Rv3779 }\end{array}$ & None & $\begin{array}{c}\text { Rv3507 } \\
\text { Rv3508 } \\
\text { Rv3514 } \\
\text { Rv3528c } \\
\text { Rv3566A }\end{array}$ & No prediction \\
\hline mcb & None & $\begin{array}{l}\text { Rv1130 } \\
\text { Rv2416c }\end{array}$ & None & $\begin{array}{c}\text { Rv3507 } \\
\text { Rv3508 } \\
\text { Rv3511 } \\
\text { Rv3514 } \\
\text { Rv3528c } \\
\text { Rv3566A } \\
\text { Rv3566c }\end{array}$ & No prediction \\
\hline mne & None & $\begin{array}{l}\text { Rv0805 } \\
\text { Rv3572 } \\
\text { Rv3779 }\end{array}$ & None & $\begin{array}{c}\text { Rv3507 } \\
\text { Rv3508 } \\
\text { Rv3511 } \\
\text { Rv3514 } \\
\text { Rv3517 } \\
\text { Rv3528c } \\
\text { Rv3566A }\end{array}$ & No prediction \\
\hline myv & None & $\begin{array}{l}\text { Rv0805 } \\
\text { Rv3572 }\end{array}$ & None & $\begin{array}{c}\text { Rv3507 } \\
\text { Rv3508 } \\
\text { Rv3511 } \\
\text { Rv3514 } \\
\text { Rv3528c }\end{array}$ & No prediction \\
\hline mye & None & $\begin{array}{c}\text { Rv0876c } \\
\text { Rv1130 } \\
\text { Rv2416c }\end{array}$ & None & $\begin{array}{c}\text { Rv3507 } \\
\text { Rv3508 } \\
\text { Rv3511 } \\
\text { Rv3517 } \\
\text { Rv3528c } \\
\text { Rv3566A } \\
\text { Rv3566c }\end{array}$ & No prediction \\
\hline mgo & None & $\begin{array}{l}\text { Rv0805 } \\
\text { Rv0876c } \\
\text { Rv3572 }\end{array}$ & None & $\begin{array}{c}\text { Rv3507 } \\
\text { Rv3508 } \\
\text { Rv3511 } \\
\text { Rv3514 } \\
\text { Rv3528c } \\
\text { Rv3566A }\end{array}$ & No prediction \\
\hline $\mathrm{mft}$ & None & Rv3572 & None & $\begin{array}{c}\text { Rv3507 } \\
\text { Rv3508 } \\
\text { Rv3511 } \\
\text { Rv3514 } \\
\text { Rv3528c }\end{array}$ & No prediction \\
\hline
\end{tabular}

2.3.1. Most of the M. Tuberculosis Complex Species Have the Ability to Catabolize Cholesterol

Among 39 MTBC species, 29 species were predicted to be positively able to catabolize cholesterol as a carbon source (Figure 4 and Table 2). There were 10 mycobacterial species, namely $M$. tuberculosis RGTB327, M. tuberculosis RGTB423, M. tuberculosis CCDC5079 (2012), M. tuberculosis CCDC5180, M. tuberculosis Erdman = ATCC 35801, M. tuberculosis CAS/NITR204, M. tuberculosis EAI5/NITR206, M. tuberculosis Haarlem/NITR202, M. bovis BCG ATCC 35743 and M. canettii CIPT 140010059, that lacked some of the cholesterol catabolic genes/proteins (Table 2), thus we did not predict their ability to catabolize cholesterol, considering that the complete cholesterol catabolic pathway had not been elucidated. 


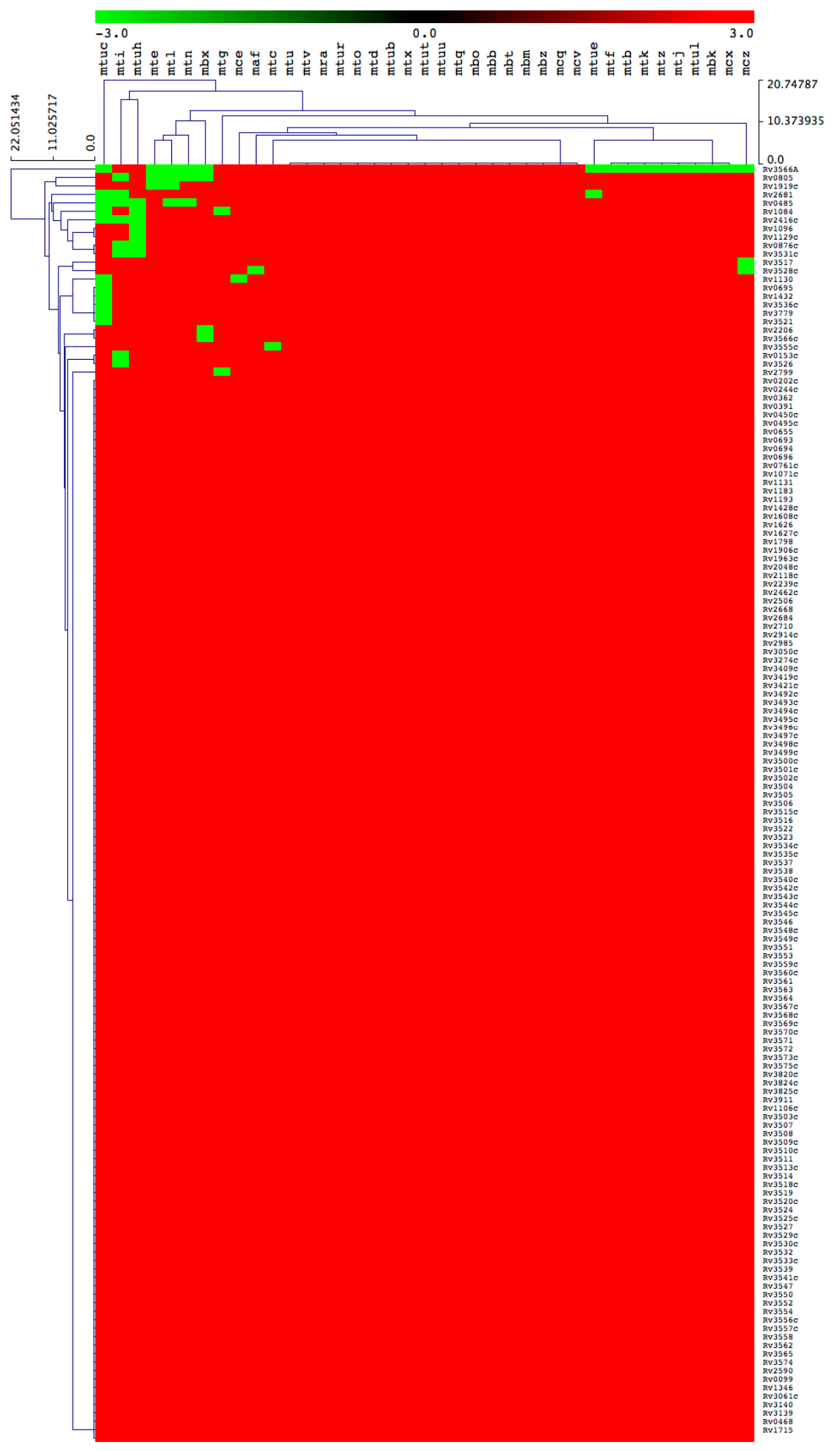

Figure 4. Heatmap of presence or absence of 151 cholesterol catabolic genes/proteins in $39 \mathrm{M}$. tuberculosis complex species. The data have been represented as -3 for gene absence (green) and 3 for gene presence (red). There are 39 mycobacterial species represented on the horizontal axis (see Table 3 for species codes) and 151 genes/proteins on the vertical axis. 
Analysis of homologous genes/proteins among MTBC species followed the same criteria as described in Section 3.3, with some exceptions for certain homologs mentioned here. For Rv0495c, homolog proteins were identified based on percentage identity, as the NCBI CDD database did not assign proteins to a particular superfamily. The percentage identity was sourced from KEGG and ranged from 99 to $100 \%$. For Rv0805, homolog proteins in M. tuberculosis RGTB423 and M. bovis BCG ATCC 35743 were not identified, as NCBI CDD did not yield any results. Furthermore, the KEGG database showed only $49 \%$ identity compared to other species' homolog proteins that showed $100 \%$ identity. Based on this, we concluded that mti and mbx did not have Rv0805 homolog(s). For Rv1432, there were no hit data for M. tuberculosis CAS/NITR204, and KEGG data revealed a different dehydrogenase hit. Thus, it was concluded that the homolog was not present. Upon review of Rv2416c, we found that the homolog protein sequence for M. tuberculosis Haarlem/NITR202 was truncated and presented as 28 amino acids compared to the other species' homologs with more than 360 amino acids. Therefore, it was decided that the homolog of Rv2416c had not been found in M. tuberculosis Haarlem/NITR202.

\subsubsection{Chelonae-Abscessus Complex Species Lack Key Cholesterol Catabolic Genes/Proteins}

All 10 MCAC species lack the homolog gene of Rv3519 from M. tuberculosis H37Rv that has been proven to be essential for survival of $M$. tuberculosis $\mathrm{H} 37 \mathrm{Rv}$ in macrophage cells and in murine infection (Figure 5 and Table 2). The function of Rv3519 is not elucidated. In addition to this, all species lack a few genes that are predicted to be essential or specifically required for growth of $M$. tuberculosis H37Rv on cholesterol (Figure 5 and Table 2). Due to the absence of key cholesterol catabolic genes/proteins in MCAC species, and considering the limited information available on cholesterol catabolism in mycobacterial species, at present we do not predict MCAC species' ability to catabolize cholesterol. Analysis of homologous genes/proteins among MCAC species followed the same criteria as described in Section 3.3, with the exception of Rv1906, as reported earlier in Section 2.3.1, where more than $40 \%$ identity to $M$. tuberculosis $\mathrm{H} 37 \mathrm{Rv}$ was taken as positive across all the categories, as the proteins were hypothetical. 


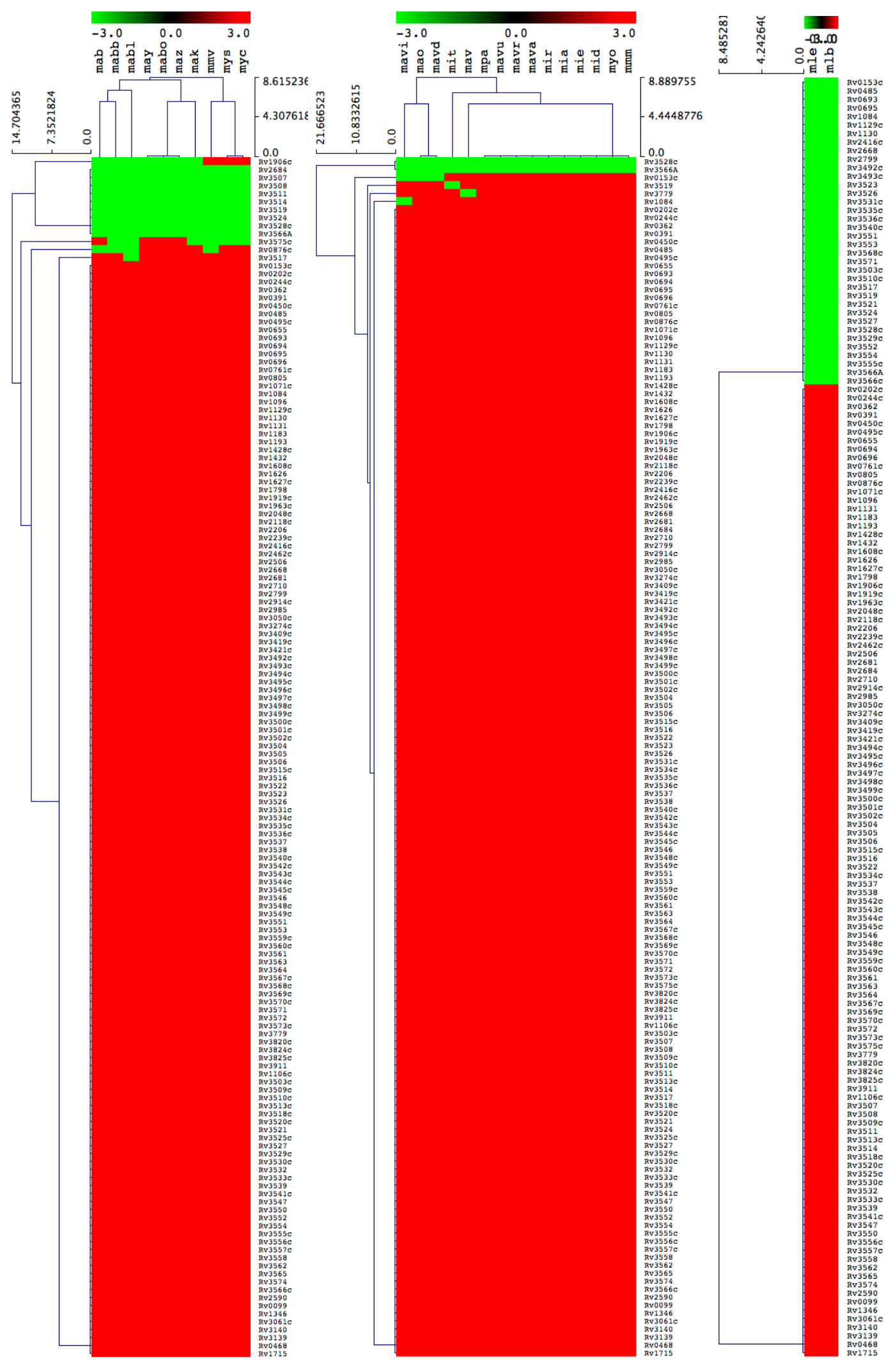

Figure 5. Heatmap of presence or absence of 151 cholesterol catabolic genes/proteins in 10 M. chelonae-abscessus complex species (left panel), 15 MAC species (center panel) and 2 Mycobacterium species causing leprosy (right panel). The data have been represented as -3 for gene absence (green) and 3 for gene presence (red). The 10, 15 and 2 mycobacterial species are represented on the horizontal axes (see Table 3 for species codes) with the 151 genes/proteins on the vertical axes. 


\subsubsection{Most of the M. Avium Complex Species Have the Ability to Catabolize Cholesterol}

Among 15 MAC species, 10 were predicted to be positive for their ability to catabolize cholesterol as a carbon source (Figure 5 and Table 2). The remaining five MAC species, M. avium subsp. paratuberculosis MAP4; M. avium subsp. paratuberculosis E1; M. avium 104; M. avium subsp. avium DJO-44271 and M. intracellulare MOTT-02, did not have the either one or two homologous genes/proteins required for growth on cholesterol (Table 2). Among 151 genes, only 6 M. tuberculosis H37Rv homologs, Rv0153c, Rv1084, Rv3779, Rv3519, Rv3528c and Rv3566A, were not found in different MAC species (Figure 5 and Table 2). Four homologs were not found in M. avium subsp. paratuberculosis E1, and two of these are predicted to be specifically required for growth on cholesterol. Since only a few genes/proteins were missing in the five species, it is difficult to predict their capability to utilize cholesterol as carbon source.

\subsubsection{Mycobacterium Causing Leprosy Species Does Not Have the Ability to Catabolize Cholesterol}

Two MCL species were predicted to be negative for their ability to catabolize cholesterol as a carbon source (Figure 5 and Table 2). Quite a large number of cholesterol catabolic genes/proteins were not found in both MCL species. Furthermore, experimental evidence proved that MCL species did not have the ability to utilize cholesterol as carbon source [44].

\subsubsection{Uncertainty about Non-Tuberculosis Mycobacterium and Saprophyte Species' Ability to} Utilize Cholesterol

Among eight NTM species, three species were predicted to be positive for cholesterol utilization as a carbon source (Figure 6 and Table 2). Of the remaining five species, M. ulcerans, M. sinense, M. kansasii 662 and M. kansasii 824 had only one missing cholesterol catabolic homolog gene/protein predicted to be essential or specifically required for M. tuberculosis H37Rv growth on cholesterol, whereas M. haemophilum had three missing cholesterol catabolic homologous genes/proteins proven to be essential (Rv3534c) and predicted to be essential or specifically required for $M$. tuberculosis $\mathrm{H} 37 \mathrm{Rv}$ growth (Rv1130 and Rv3534c) on cholesterol (Figure 6 and Table 2). Because of the absence of only a few genes/proteins, it is difficult to predict the five NTM species' cholesterol utilization ability as a carbon source.

In the SAP species, Mycobacterium sp. JS623 (msa) and M. fortuitum (mft) lacked a single homologous gene/protein, and the other SAP species had more than one missing cholesterol catabolic homologous gene/protein predicted to be essential or specifically required for $M$. tuberculosis H37Rv growth on cholesterol (Figure 6 and Table 2). However, considering the contrasting lifestyle and habitat of SAP species compared to M. tuberculosis H37Rv, the role of cholesterol catabolic genes/proteins proven to be or predicted to be essential for survival of $M$. tuberculosis in macrophage cells and in murine infection [19] that were not found in SAP species may indicate that these genes/proteins do not play any role in cholesterol utilization by SAP species, and possibly all SAPs can utilize cholesterol as a carbon source. The latest study by Guo et al. [45] strongly supports this argument where quite a number of saprophytes, including M. vanbaalenii, have been shown to degrade cholesterol. However, experimental evidence will shed more light on SAP species' ability to metabolize cholesterol. For this reason, we did not predict SAP species' ability to utilize cholesterol as carbon source. 


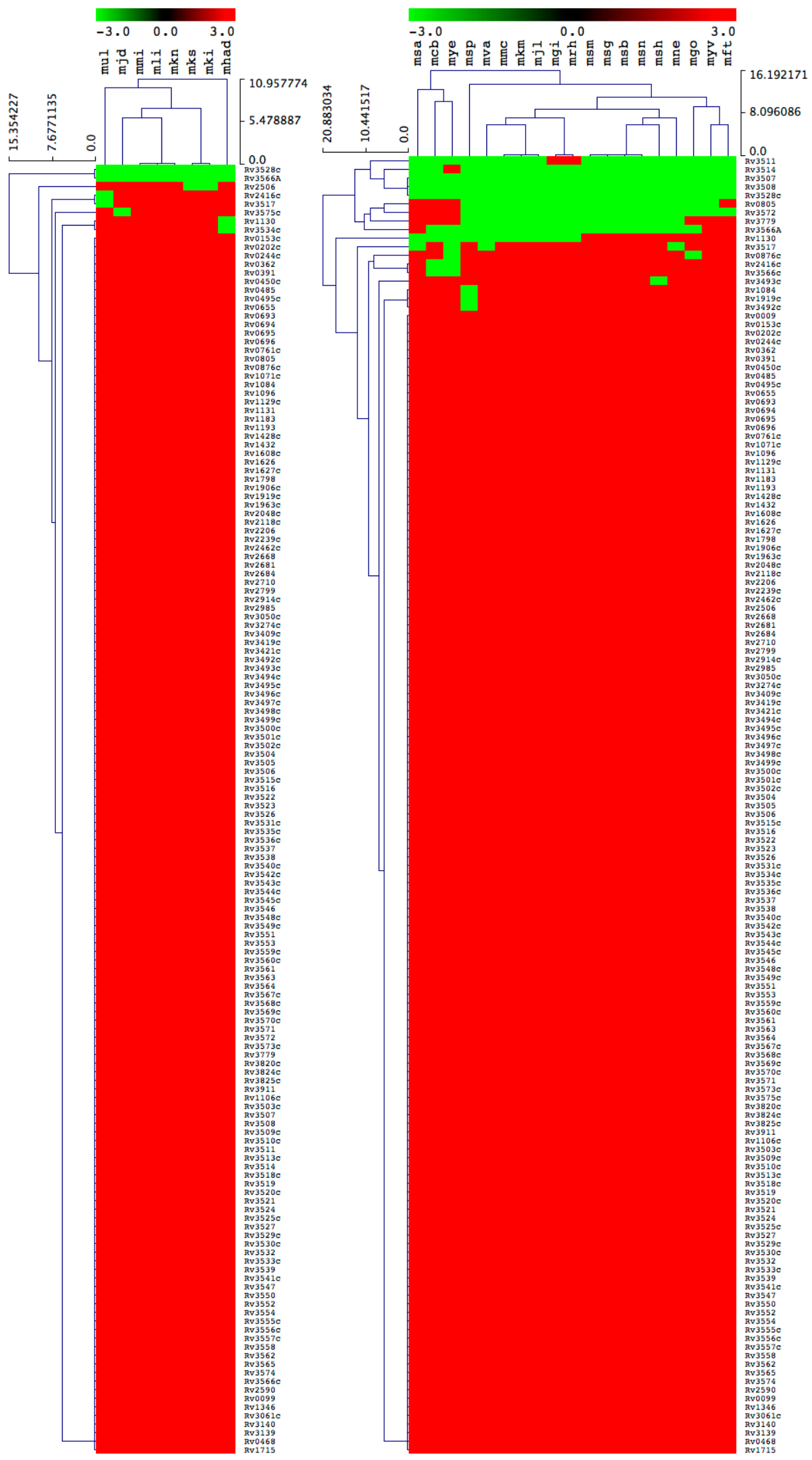

Figure 6. Heatmap of presence or absence of 151 cholesterol catabolic genes/proteins in 8 non-tuberculosis Mycobacterium species (left panel) and 19 SAP (right panel). The data have been represented as -3 for gene absence (green) and 3 for gene presence (red). The 8 and 19 mycobacterial species are represented on the horizontal axes (see Table 3 for species codes) with the 151 genes/proteins on the vertical axes. 


\section{Materials and Methods}

\subsection{Species and Database}

In total 93 mycobacterial species belonging to 6 different categories were used in this study (Table 3). The 6 categories included M. tuberculosis complex (MTBC) (39 species), M. chelonae-abscessus complex (MCAC) (10 species), M. avium complex (MAC) (15 species), mycobacteria causing leprosy (MCL) (2 species), non-tuberculous mycobacteria (NTM) (8 species) and saprophytes (SAP) (19 species). The criteria for separation of the mycobacterial species into six different groups were based on their characteristic features, including ecological niches, as well as the nature and site of infection as described elsewhere $[46,47]$. Taxonomical grouping of mycobacterial species was also taken into consideration, as described elsewhere [48]. Detailed information on species, their categories and genome database links are listed in Table 3.

\subsection{Cholesterol Catabolism}

Published research and review articles [19,22-27] were consulted to create a schematic diagram of the cholesterol catabolic pathway of M. tuberculosis H37Rv, showing the intermediate metabolites and the enzymes involved in different reactions. According to Ouellet et al. [19], the cholesterol catabolic pathway of $M$. tuberculosis can be divided into two major phases-firstly, the initial degradation of the aliphatic side chain, and then the subsequent degradation of the A-D rings. In this study, the two phases were drawn up separately using ChemDraw software [49].

\subsection{Cholesterol Catabolic Genes/Proteins Analysis in Mycobacterial Species}

In total, 152 genes/proteins identified in the study as part of the cholesterol catabolic pathway in M. tuberculosis H37Rv. These were selected for comparative analysis from 92 mycobacterial species. The selected 152 protein sequences were retrieved from the Kyoto Encyclopedia of Genes and Genomes (KEGG) database, using their respective gene codes.

\subsubsection{BLAST Analysis}

The protein sequences of $152 \mathrm{M}$. tuberculosis $\mathrm{H} 37 \mathrm{Rv}$ proteins were copied and pasted into the Basic Local Alignment Search Tool (BLAST) in the KEGG database (http:/ /www.genome.jp/tools/blast/). The amino acid sequence was entered in the "sequence data" field, then "favorite organism code or category" was selected under the "KEGG GENES" button, "Mycobacterium" was entered in the free text field provided and the "compute" link was selected at the top. Once the BLAST was complete, the "show all results" link was selected. The resulting output was copied and pasted into an Excel program to extract the required data (organism code, enzyme code, enzyme name, identity and homology (positives)) from all of the BLAST output data, which were then tabulated under each organism name and code (Supplementary Dataset 1).

\subsubsection{Excel Program for Extracting KEGG BLAST Data}

To extract the required data from the BLAST output data obtained from the KEGG database, an Excel program written in an Excel worksheet was used. The generated program is presented in the Supplementary Materials.

\subsubsection{Data Collection and Protein Domain/Function Analysis}

All the top hit protein sequences in 92 mycobacterial species were collected (Supplementary Dataset 2) and input into the National Center for Biotechnology Information Batch Web CD-search Tool (NCBI CDD) [50]. Based on the NCBI CDD results, proteins belonging to the same family/superfamily were identified (Supplementary Dataset 3). For some proteins, no results were obtained with the NCBI 
CDD. Thus, the KEGG database was searched for possible functions or domains to determine whether they belonged to the same group (Supplementary Dataset 1).

3.3.4. Assessing the Presence or Absence of Cholesterol Catabolic Gene/Protein Homologs in Mycobacterial Species

The superfamilies, as per the NCBI CDD output, were considered to determine whether the genes/proteins from the 92 mycobacterial species matched those from M. tuberculosis H37Rv. If no data on superfamilies were available in the NCBI database, a secondary review was performed of the KEGG BLAST output data by looking at the percentage identity, percentage homology and name (and thus also the function) of each of the genes/proteins. However, the presence or absence of some proteins in different mycobacterial species was determined based on the information below.

The Rv3512 gene/protein homolog was not identified in many species in the KEGG BLAST output. This may have been due to annotation errors, as M. tuberculosis H37Rv (1998) (mtu) and M. tuberculosis H37Rv (2012) (mtv) showed different results. Furthermore, this gene is not shown to be essential for cholesterol catabolism. Thus, this gene was omitted from the analysis.

For Rv1906, more than $40 \%$ identity to $M$. tuberculosis H37Rv was taken as positive across all categories, as the proteins are hypothetical. According to this, the negative species were M. abscessus ATCC 19977, M. abscessus subsp. bolletii 50594, M. abscessus subsp. bolletii GO 06, M. abscessus subsp. bolletii MA 1948, M. abscessus subsp. bolletii MC1518, M. abscessus subsp. bolletii CCUG 48898 = JCM 15300, M. abscessus subsp. bolletii 103, M. abscessus subsp. abscessus MM1513, M. abscessus DJO-44274 and $M$. abscessus 4529 .

For Rv3566A, Rv3527 and Rv3572, more than $40 \%$ identity to M. tuberculosis H37Rv was taken as positive across all categories, as the proteins are hypothetical.

The results were tabulated per complex by colour-coding the cells according to the following criteria: red $=$ gene homolog present; green $=$ gene homolog not found .

\subsection{Generation of Gene/Protein Heatmaps}

The presence or absence of genes/proteins in mycobacterial species was shown with heatmaps following the method described elsewhere [51]. Briefly, the data were represented as -3 for gene absence (green) and 3 for gene presence (red). A tab-delimited file was imported into a Multi-Experiment Viewer (Mev) [52]. A Euclidean distance metric was used to perform hierarchical clustering. Mycobacterial species are presented on the horizontal axis (see Supplementary Dataset 4 for codes) and the 151 genes on the vertical axis. 
Table 3. List of mycobacterial species and their database links used in the study. For some species, references were not available despite the genome database being available for public use at the Kyoto Encyclopedia of Genes and Genomes (KEGG) database [53] and thus references were not cited for these.

\begin{tabular}{|c|c|c|c|}
\hline Species Name & Organism Code & Database Link & Reference \\
\hline \multicolumn{4}{|l|}{ Mycobacterium tuberculosis complex (MTBC) } \\
\hline Mycobacterium tuberculosis $\mathrm{H} 37 \mathrm{Rv}$ & mtu & http://www.genome.jp/kegg-bin/show_organism?org=mtu & [54] \\
\hline Mycobacterium tuberculosis $\mathrm{H} 37 \mathrm{Rv}$ & $\mathrm{mtv}$ & http://www.genome.jp/kegg-bin/show_organism?org=mtv & \\
\hline Mycobacterium tuberculosis CDC1551 & mtc & http://www.genome.jp/kegg-bin/show_organism?org=mtc & [55] \\
\hline Mycobacterium tuberculosis H37Ra & mra & http://www.genome.jp/kegg-bin/show_organism?org=mra & [56] \\
\hline Mycobacterium tuberculosis F11 & $\mathrm{mtf}$ & http://www.genome.jp/kegg-bin/show_organism?org=mtf & \\
\hline Mycobacterium tuberculosis KZN 1435 & $\mathrm{mtb}$ & http://www.genome.jp/kegg-bin/show_organism?org=mtb & \\
\hline Mycobacterium tuberculosis KZN 4207 & $\mathrm{mtk}$ & http://www.genome.jp/kegg-bin/show_organism?org=mtk & \\
\hline Mycobacterium tuberculosis KZN 605 & $\mathrm{mtz}$ & http://www.genome.jp/kegg-bin/show_organism?org=mtz & \\
\hline Mycobacterium tuberculosis RGTB327 & $\mathrm{mtg}$ & http://www.genome.jp/kegg-bin/show_organism?org=mtg & [57] \\
\hline Mycobacterium tuberculosis RGTB423 & $\mathrm{mti}$ & http://www.genome.jp/kegg-bin/show_organism?org=mti & [57] \\
\hline Mycobacterium tuberculosis CCDC5079 & mte & http://www.genome.jp/kegg-bin/show_organism?org=mte & [58] \\
\hline Mycobacterium tuberculosis CCDC5079 & mtur & http://www.genome.jp/kegg-bin/show_organism?org=mtur & [59] \\
\hline Mycobacterium tuberculosis CCDC5180 & $\mathrm{mtl}$ & http://www.genome.jp/kegg-bin/show_organism?org=mtl & [58] \\
\hline Mycobacterium tuberculosis CTRI-2 & mto & http://www.genome.jp/kegg-bin/show_organism?org=mto & [60] \\
\hline Mycobacterium tuberculosis UT205 & $\mathrm{mtd}$ & http://www.genome.jp/kegg-bin/show_organism?org=mtd & [61] \\
\hline Mycobacterium tuberculosis Erdman = ATCC 35801 & $\mathrm{mtn}$ & http://www.genome.jp/kegg-bin/show_organism?org=mtn & [62] \\
\hline Mycobacterium tuberculosis Beijing/NITR203 & $\mathrm{mtj}$ & http://www.genome.jp/kegg-bin/show_organism?org=mtj & [63] \\
\hline Mycobacterium tuberculosis 7199-99 & mtub & http://www.genome.jp/kegg-bin/show_organism?org=mtub & [64] \\
\hline Mycobacterium tuberculosis CAS/NITR204 & mtuc & http://www.genome.jp/kegg-bin/show_organism?org=mtuc & [63] \\
\hline Mycobacterium tuberculosis EAI5/NITR206 & mtue & http://www.genome.jp/kegg-bin/show_organism?org=mtue & [63] \\
\hline Mycobacterium tuberculosis EAI5 & $\mathrm{mtx}$ & http://www.genome.jp/kegg-bin/show_organism?org=mtx & [65] \\
\hline Mycobacterium tuberculosis Haarlem/NITR202 & mtuh & http://www.genome.jp/kegg-bin/show_organism?org=mtuh & [63] \\
\hline Mycobacterium tuberculosis Haarlem & mtul & http://www.genome.jp/kegg-bin/show_organism?org=mtul & \\
\hline Mycobacterium tuberculosis BT1 & mtut & http://www.genome.jp/kegg-bin/show_organism?org=mtut & \\
\hline Mycobacterium tuberculosis BT2 & mtuu & http://www.genome.jp/kegg-bin/show_organism?org=mtuu & \\
\hline Mycobacterium tuberculosis HKBS1 & $\mathrm{mtq}$ & http://www.genome.jp/kegg-bin/show_organism?org=mtq & \\
\hline Mycobacterium bovis AF2122/97 & mbo & http://www.genome.jp/kegg-bin/show_organism?org=mbo & [66] \\
\hline
\end{tabular}


Table 3. Cont

\begin{tabular}{|c|c|c|c|}
\hline Species Name & Organism Code & Database Link & Reference \\
\hline Mycobacterium bovis BCG Pasteur 1173P2 & $\mathrm{mbb}$ & http://www.genome.jp/kegg-bin/show_organism?org=mbb & [67] \\
\hline Mycobacterium bovis BCG Tokyo 172 & $\mathrm{mbt}$ & http://www.genome.jp/kegg-bin/show_organism?org=mbt & [68] \\
\hline Mycobacterium bovis BCG Mexico & $\mathrm{mbm}$ & http://www.genome.jp/kegg-bin/show_organism?org=mbm & [69] \\
\hline Mycobacterium bovis BCG Korea 1168P & mbk & http://www.genome.jp/kegg-bin/show_organism?org=mbk & [70] \\
\hline Mycobacterium bovis BCG ATCC 35743 & $\mathrm{mbx}$ & http://www.genome.jp/kegg-bin/show_organism?org=mbx & [71] \\
\hline Mycobacterium bovis ATCC BAA-935 & $\mathrm{mbz}$ & http://www.genome.jp/kegg-bin/show_organism?org=mbz & \\
\hline Mycobacterium africanum & maf & http://www.genome.jp/kegg-bin/show_organism?org=maf & [72] \\
\hline Mycobacterium canettii CIPT 140010059 & mce & http://www.genome.jp/kegg-bin/show_organism?org=mce & [72] \\
\hline Mycobacterium canettii CIPT 140060008 & mcq & http://www.genome.jp/kegg-bin/show_organism?org=mcq & [73] \\
\hline Mycobacterium canettii CIPT 140070008 & mcv & http://www.genome.jp/kegg-bin/show_organism?org=mcv & [73] \\
\hline Mycobacterium canettii CIPT 140070010 & $\operatorname{mcx}$ & http://www.genome.jp/kegg-bin/show_organism?org=mcx & [73] \\
\hline Mycobacterium canettii CIPT 140070017 & $\mathrm{mcz}$ & http://www.genome.jp/kegg-bin/show_organism?org=mcz & [73] \\
\hline \multicolumn{4}{|l|}{ Mycobacteria causing leprosy (MCL) } \\
\hline Mycobacterium leprae TN & mle & http://www.genome.jp/kegg-bin/show_organism?org=mle & [74] \\
\hline Mycobacterium leprae $\mathrm{Br} 4923$ & $\mathrm{mlb}$ & http://www.genome.jp/kegg-bin/show_organism?org=mlb & [75] \\
\hline \multicolumn{4}{|l|}{ Mycobacterium avium complex (MAC) } \\
\hline Mycobacterium avium subsp. paratuberculosis K-10 & mpa & http://www.genome.jp/kegg-bin/show_organism?org=mpa & [76] \\
\hline Mycobacterium avium subsp. paratuberculosis MAP4 & mao & http://www.genome.jp/kegg-bin/show_organism?org=mao & [77] \\
\hline Mycobacterium avium subsp. paratuberculosis E1 & mavi & http://www.genome.jp/kegg-bin/show_organism?org=mavi & [78] \\
\hline Mycobacterium avium subsp. paratuberculosis E93 & mavu & http://www.genome.jp/kegg-bin/show_organism?org=mavu & [78] \\
\hline Mycobacterium avium 104 & mav & http://www.genome.jp/kegg-bin/show_organism?org=mav & \\
\hline Mycobacterium avium subsp. avium DJO-44271 & mavd & http://www.genome.jp/kegg-bin/show_organism?org=mavd & \\
\hline Mycobacterium avium subsp. avium $2285(\mathrm{R})$ & mavr & http://www.genome.jp/kegg-bin/show_organism?org=mavr & \\
\hline Mycobacterium avium subsp. avium 2285 (S) & mava & http://www.genome.jp/kegg-bin/show_organism?org=mava & \\
\hline Mycobacterium intracellulare MOTT-02 & mit & http://www.genome.jp/kegg-bin/show_organism?org=mit & [79] \\
\hline Mycobacterium intracellulare MOTT-64 & $\operatorname{mir}$ & http://www.genome.jp/kegg-bin/show_organism?org=mir & [80] \\
\hline Mycobacterium intracellulare ATCC 13950 & mia & http://www.genome.jp/kegg-bin/show_organism?org=mia & [81] \\
\hline Mycobacterium intracellulare 1956 & mie & http://www.genome.jp/kegg-bin/show_organism?org=mie & \\
\hline Mycobacterium indicus pranii & mid & http://www.genome.jp/kegg-bin/show_organism?org=mid & [82] \\
\hline Mycobacterium yongonense & myo & http://www.genome.jp/kegg-bin/show_organism?org=myo & [83] \\
\hline Mycobacterium sp. МОTТ36Y & $\mathrm{mmm}$ & http://www.genome.jp/kegg-bin/show_organism?org=mmm & [84] \\
\hline
\end{tabular}


Table 3. Cont

\begin{tabular}{|c|c|c|c|}
\hline Species Name & Organism Code & Database Link & Reference \\
\hline \multicolumn{4}{|l|}{ Saprophytes (SAP) } \\
\hline Mycobacterium smegmatis MC2 155 & $\mathrm{msm}$ & http://www.genome.jp/kegg-bin/show_organism?org=msm & \\
\hline Mycobacterium smegmatis MC2 155 & msg & http://www.genome.jp/kegg-bin/show_organism?org=msg & [85] \\
\hline Mycobacterium smegmatis MC2 155 & $\mathrm{msb}$ & http://www.genome.jp/kegg-bin/show_organism?org=msb & [86] \\
\hline Mycobacterium smegmatis INHR1 & $\mathrm{msn}$ & http://www.genome.jp/kegg-bin/show_organism?org=msn & [87] \\
\hline Mycobacterium smegmatis INHR2 & $\mathrm{msh}$ & http://www.genome.jp/kegg-bin/show_organism?org=msh & [86] \\
\hline Mycobacterium sp. JS623 & msa & http://www.genome.jp/kegg-bin/show_organism?org=msa & \\
\hline Mycobacterium vanbaalenii & mva & http://www.genome.jp/kegg-bin/show_organism?org=mva & \\
\hline Mycobacterium gilvum PYR-GCK & mgi & http://www.genome.jp/kegg-bin/show_organism?org=mgi & \\
\hline Mycobacterium gilvum Spyr1 & $\mathrm{msp}$ & http://www.genome.jp/kegg-bin/show_organism?org=msp & [87] \\
\hline Mycobacterium sp. MCS & $\mathrm{mmc}$ & http://www.genome.jp/kegg-bin/show_organism?org=mmc & \\
\hline Mycobacterium sp. KMS & $\mathrm{mkm}$ & http://www.genome.jp/kegg-bin/show_organism?org=mkm & \\
\hline Mycobacterium sp. JLS & mjl & http://www.genome.jp/kegg-bin/show_organism?org=mjl & \\
\hline Mycobacterium rhodesiae & mrh & http://www.genome.jp/kegg-bin/show_organism?org=mrh & \\
\hline Mycobacterium chubuense & $\mathrm{mcb}$ & http://www.genome.jp/kegg-bin/show_organism?org=mcb & \\
\hline Mycobacterium neoaurum & mne & http://www.genome.jp/kegg-bin/show_organism?org=mne & [88] \\
\hline Mycobacterium sp. VKM Ac-1817D & myv & http://www.genome.jp/kegg-bin/show_organism?org=myv & [88] \\
\hline Mycobacterium sp. EPa45 & mye & http://www.genome.jp/kegg-bin/show_organism?org=mye & [89] \\
\hline Mycobacterium goodii & mgo & http://www.genome.jp/kegg-bin/show_organism?org=mgo & [90] \\
\hline Mycobacterium fortuitum & $\mathrm{mft}$ & http://www.genome.jp/kegg-bin/show_organism?org=mft & [91] \\
\hline \multicolumn{4}{|l|}{ Non-tuberculosis mycobacteria (NTM) } \\
\hline Mycobacterium ulcerans & mul & http://www.genome.jp/kegg-bin/show_organism?org=mul & [92] \\
\hline Mycobacterium sinense & mjd & http://www.genome.jp/kegg-bin/show_organism?org=mjd & [93] \\
\hline Mycobacterium marinum & $\mathrm{mmi}$ & http://www.genome.jp/kegg-bin/show_organism?org=mmi & [94] \\
\hline Mycobacterium liflandii & mli & http://www.genome.jp/kegg-bin/show_organism?org=mli & [95] \\
\hline Mycobacterium kansasii ATCC 12478 & mkn & http://www.genome.jp/kegg-bin/show_organism?org=mkn & \\
\hline Mycobacterium kansasii 662 & mks & http://www.genome.jp/kegg-bin/show_organism?org=mks & \\
\hline Mycobacterium kansasii 824 & mki & http://www.genome.jp/kegg-bin/show_organism?org=mki & \\
\hline Mycobacterium haemophilum & mhad & http://www.genome.jp/kegg-bin/show_organism?org=mhad & [96] \\
\hline
\end{tabular}


Table 3. Cont

\begin{tabular}{|c|c|c|c|}
\hline Species Name & Organism Code & Database Link & Reference \\
\hline \multicolumn{4}{|l|}{ Mycobacterium chelonae-abscessus complex (MCAC) } \\
\hline Mycobacterium abscessus ATCC 19977 & mab & http://www.genome.jp/kegg-bin/show_organism?org=mab & [97] \\
\hline Mycobacterium abscessus subsp. bolletii 50594 & mabb & http://www.genome.jp/kegg-bin/show_organism?org=mabb & [98] \\
\hline Mycobacterium abscessus subsp. bolletii $\mathrm{GO} 06$ & $\mathrm{mmv}$ & http://www.genome.jp/kegg-bin/show_organism?org=mmv & [99] \\
\hline Mycobacterium abscessus subsp. bolletii MA 1948 & may & http://www.genome.jp/kegg-bin/show_organism?org=may & \\
\hline Mycobacterium abscessus subsp. bolletii MC1518 & mabo & http://www.genome.jp/kegg-bin/show_organism?org=mabo & \\
\hline Mycobacterium abscessus subsp. bolletii CCUG $48898=$ JCM 15300 & mabl & http://www.genome.jp/kegg-bin/show_organism?org=mabl & [100] \\
\hline Mycobacterium abscessus subsp. bolletii 103 & maz & http://www.genome.jp/kegg-bin/show_organism?org=maz & \\
\hline Mycobacterium abscessus subsp. abscessus & mak & http://www.genome.jp/kegg-bin/show_organism?org=mak & \\
\hline Mycobacterium abscessus DJO-44274 & mys & http://www.genome.jp/kegg-bin/show_organism?org=mys & \\
\hline Mycobacterium abscessus 4529 & myc & http://www.genome.jp/kegg-bin/show_organism?org=myc & \\
\hline
\end{tabular}




\section{Conclusions}

The study results were intended to predict mycobacterial species' ability to utilize cholesterol as a carbon source. To achieve this task, a comprehensive cholesterol catabolic pathway was deduced from the available literature. Genes/proteins involved in the cholesterol catabolism were identified, and comprehensive comparative analysis of $M$. tuberculosis $\mathrm{H} 37 \mathrm{Rv}$ homologous genes/proteins in different mycobacterial species was performed, using a newly developed software tool to extract homologous protein data. Gene/protein sequences were collected and subjected to protein family assignment and functional analysis. Finally, based on the presence of genes/proteins critical for cholesterol catabolism, mycobacterial species' ability to catabolize cholesterol was determined. There are certain points to be taken from the study on predicting the cholesterol utilization capability of mycobacterial species belonging to categories such as MAC, SAP and NTM-i.e., that most of the homolog cholesterol catabolic genes/proteins missing from these species have in fact been proven to be essential for survival of $M$. tuberculosis $\mathrm{H} 37 \mathrm{Rv}$ in macrophage cells and in murine infection, but the number of these missing genes/proteins is limited to a single gene in most cases. Thus, it is difficult to predict the cholesterol utilization ability for MAC and NTM species. It is not clear whether these genes/proteins play any role in cholesterol assimilation in SAP species, since these species have different lifestyle and habitat properties compared to $M$. tuberculosis H37Rv. Overall, this study opened new vistas on comparative analysis of cholesterol catabolic genes/proteins in mycobacterial species, and study results should be taken as a source of information on cholesterol catabolic genes/proteins in mycobacterial species.

Supplementary Materials: Supplementary materials can be found at http:/ / www.mdpi.com/1422-0067/20/5/ 1032/s1.

Author Contributions: Conceptualization, K.S.; Data curation, R.v.W., M.v.W., S.S.M., D.R.N. and K.S.; Formal analysis, R.v.W., M.v.W., S.S.M., D.R.N. and K.S.; Funding acquisition, K.S.; Investigation, R.v.W., M.v.W., S.S.M., D.R.N. and K.S.; Methodology, R.v.W., M.v.W., S.S.M., D.R.N. and K.S.; Project administration, K.S.; Resources, K.S.; Supervision, S.S.M. and K.S.; Validation, R.v.W., M.v.W., S.S.M., D.R.N. and K.S.; Visualization, K.S.; Writing—original draft, R.v.W., M.v.W., S.S.M., D.R.N. and K.S.; Writing—review \& editing, K.S.

Funding: R.v.W. thanks the National Research Foundation (NRF), South Africa for DST-NRF Scarce-Skills Master's Scholarship (Grant No. 107924). M.v.W. and R.v.W. express their sincere gratitude to the Central University of Technology for master's (R.v.W.) and doctoral bursaries (M.v.W.). K.S. expresses sincere gratitude to the University of Zululand Research Committee for funding (Grant No. C686) and to the NRF, South Africa for competitive support grant (Grant No. 114159).

Acknowledgments: The authors want to thank Barbara Bradley, Pretoria, South Africa for English language editing.

Conflicts of Interest: The authors declare no conflict of interest. The founding sponsors had no role in the design of the study, the collection, analysis or interpretation of data, the writing of the manuscript or the decision to publish the results.

\section{References}

1. World Health Organization (WHO). Available online: https://www.who.int/tb/publications/global_ report/en/ (accessed on 15 January 2019).

2. Sotgiu, G.; Sulis, G.; Matteelli, A. Tuberculosis-A World Health Organization Perspective. Microbiol. Spectr. 2017, 5, 1-2. [CrossRef] [PubMed]

3. World Health Organization (WHO). Available online: http://www.who.int/tb/publications/global_report/ gtbr15_main_text.pdf. (accessed on 15 January 2019).

4. Glickman, M.S.; Jacobs, W.R. Microbial pathogenesis of Mycobacterium tuberculosis: Dawn of a discipline. Cell 2001, 104, 477-485. [CrossRef]

5. Flynn, J.L.; Chan, J. Tuberculosis: Latency and reactivation. Infect. Immun. 2001, 69, 4195-4201. [CrossRef] [PubMed]

6. Migliori, G.B.; Centis, R.; D’Ambrosio, L.; Spanevello, A.; Borroni, E.; Cirillo, D.M.; Sotgiu, G. Totally drug-resistant and extremely drug-resistant tuberculosis: The same disease? Clin. Infect. Dis. 2012, 54, 379-1380. [CrossRef] [PubMed] 
7. Clark-Curtiss, J.E.; Haydel, S.E. Molecular genetics of Mycobacterium tuberculosis pathogenesis. Annu. Rev. Microbiol. 2003, 57, 517-549. [CrossRef] [PubMed]

8. Koul, A.; Herget, T.; Klebl, B.; Ullrich, A. Interplay between mycobacteria and host signalling pathways. Nat. Rev. Microbiol. 2004, 2, 189-202. [CrossRef] [PubMed]

9. Kim, M.J.; Wainwright, H.C.; Locketz, M.; Bekker, L.G.; Walther, G.B.; Dittrich, C.; Visser, A.; Wang, W.; Hsu, F.F.; Wiehart, U.; et al. Caseation of human tuberculosis granulomas correlates with elevated host lipid metabolism. EMBO Mol. Med. 2010, 2, 258-274. [CrossRef] [PubMed]

10. Schäfer, G.; Guler, R.; Murray, G.; Brombacher, F.; Brown, G.D. The role of scavenger receptor B1 in infection with Mycobacterium tuberculosis in a murine model. PLoS ONE 2009, 4, e8448. [CrossRef] [PubMed]

11. Martens, G.W.; Arikan, M.C.; Lee, J.; Ren, F.; Vallerskog, T.; Kornfeld, H. Hypercholesterolemia impairs immunity to tuberculosis. Infect. Immun. 2008, 76, 3464-3472. [CrossRef] [PubMed]

12. Gatfield, J.; Pieters, J. Essential role for cholesterol in entry of mycobacteria into macrophages. Science 2000, 288, 1647-1651. [CrossRef] [PubMed]

13. Peyron, P.; Bordier, C.; Elsa-Noah, N.; Maridonneau-Parini, I. Nonopsonic phagocytosis of Mycobacterium kansasii by human neutrophils depends on cholesterol and is mediated by CR3 associated with glycosylphosphatidylinositol-anchored proteins. J. Immunol. 2000, 165, 5186-5191. [CrossRef] [PubMed]

14. Muñoz, S.; Rivas-Santiago, B.; Enciso, J.A. Mycobacterium tuberculosis entry into mast cells through cholesterol-rich membrane microdomains. Scand. J. Immunol. 2009, 70, 256-263. [CrossRef] [PubMed]

15. Pieters, J.; Gatfield, J. Hijacking the host; survival of pathogenic mycobacteria inside macrophages. Trends Microbiol. 2002, 10, 142-146. [CrossRef]

16. Nguyen, L.; Pieters, J. The Trojan horse: Survival tactics of pathogenic mycobacteria in macrophages. Trends Cell Biol. 2005, 15, 269-276. [CrossRef] [PubMed]

17. Pandey, A.K.; Sassetti, C.M. Mycobacterial persistence requires the utilization of host cholesterol. Proc. Natl. Acad. Sci. USA 2008, 105, 4376-4380. [CrossRef] [PubMed]

18. De Chastellier, C.; Thilo, L. Cholesterol depletion in Mycobacterium avium-infected macrophages overcomes the block in phagosome maturation and leads to the reversible sequestration of viable mycobacteria in phagolysosome-derived autophagic vacuoles. Cell. Microbiol. 2006, 8, 242-256. [CrossRef] [PubMed]

19. Ouellet, H.; Johnston, J.B.; de Montellano, P.R.O. Cholesterol catabolism as a therapeutic target in Mycobacterium tuberculosis. Trends Microbiol. 2011, 19, 530-539. [CrossRef] [PubMed]

20. García, J.L.; Uhía, I.; Galán, B. Catabolism and biotechnological applications of cholesterol degrading bacteria. Microb. Biotechnol. 2012, 5, 679-699. [CrossRef] [PubMed]

21. Dubnau, E.; Chan, J.; Mohan, V.P.; Smith, I. Responses of Mycobacterium tuberculosis to growth in the mouse lung. Infect. Immun. 2005, 73, 3754-3757. [CrossRef] [PubMed]

22. Nesbitt, N.M.; Yang, X.; Fontán, P.; Kolesnikova, I.; Smith, I.; Sampson, N.S.; Dubnau, E. A thiolase of Mycobacterium tuberculosis is required for virulence and production of androstenedione and androstadienedione from cholesterol. Infect. Immun. 2010, 78, 275-282. [CrossRef] [PubMed]

23. Thomas, S.T.; VanderVen, B.C.; Sherman, D.R.; Russell, D.G.; Sampson, N.S. Pathway profiling in Mycobacterium tuberculosis elucidation of cholesterol-derived catabolite and enzymes that catalyze its metabolism. J. Biol. Chem. 2011, 286, 43668-43678. [CrossRef] [PubMed]

24. Świzdor, A.; Panek, A.; Milecka-Tronina, N.; Kołek, T. Biotransformations utilizing $\beta$-oxidation cycle reactions in the synthesis of natural compounds and medicines. Int. J. Mol. Sci. 2012, 13, 16514-16543. [CrossRef] [PubMed]

25. Van der Geize, R.; Yam, K.; Heuser, T.; Wilbrink, M.H.; Hara, H.; Anderton, M.C.; Sim, E.; Dijkhuizen, L.; Davies, J.E.; Mohn, W.W.; et al. A gene cluster encoding cholesterol catabolism in a soil actinomycete provides insight into Mycobacterium tuberculosis survival in macrophages. Proc. Natl. Acad. Sci. USA 2007, 104, 1947-1952. [CrossRef] [PubMed]

26. Griffin, J.E.; Gawronski, J.D.; DeJesus, M.A.; Loerger, T.R.; Akerley, B.J.; Sassetti, C.M. High-resolution phenotypic profiling defines genes essential for mycobacterial growth and cholesterol catabolism. PLoS Pathol. 2011, 7, e1002251. [CrossRef] [PubMed]

27. Crowe, A.M.; Casabon, I.; Brown, K.L.; Liu, J.; Lian, J.; Rogalski, J.C.; Hurst, T.E.; Snieckus, V.; Foster, L.J.; Eltis, L.D. Catabolism of the last two steroid rings in Mycobacterium tuberculosis and other bacteria. mBio 2017, 8, e00321-17. [CrossRef] [PubMed] 
28. Rosłoniec, K.Z.; Wilbrink, M.H.; Capyk, J.K.; Mohn, W.W.; Ostendorf, M.; Van Der Geize, R.; Dijkhuizen, L.; Eltis, L.D. Cytochrome P450 125 (CYP125) catalyses C26-hydroxylation to initiate sterol side-chain degradation in Rhodococcus jostii RHA1. Mol. Microbiol. 2009, 74, 1031-1043. [CrossRef] [PubMed]

29. Johnston, J.B.; Ouellet, H.; de Montellano, P.R.O. Functional redundancy of steroid C26-monooxygenase activity in Mycobacterium tuberculosis revealed by biochemical and genetic analyses. J. Biol. Chem. 2010, 285, 36352-36360. [CrossRef] [PubMed]

30. García-Fernández, E.; Frank, D.J.; Galán, B.; Kells, P.M.; Podust, L.M.; García, J.L.; Ortiz de Montellano, P.R. A highly conserved mycobacterial cholesterol catabolic pathway. Environ. Microbiol. 2013, 15, 2342-2359. [CrossRef] [PubMed]

31. Wipperman, M.F.; Yang, M.; Thomas, S.T.; Sampson, N.S. Shrinking the FadE proteome of Mycobacterium tuberculosis: Insights into cholesterol metabolism through identification of an $\alpha 2 \beta 2$ heterotetrameric acyl coenzyme A dehydrogenase family. J. Bacteriol. 2013, 195, 4331-4341. [CrossRef] [PubMed]

32. Schnappinger, D.; Ehrt, S.; Voskuil, M.I.; Liu, Y.; Mangan, J.A.; Monahan, I.M.; Dolganov, G.; Efron, B.; Butcher, P.D.; Nathan, C.; et al. Transcriptional adaptation of Mycobacterium tuberculosis within macrophages. J. Exp. Med. 2003, 198, 693-704. [CrossRef] [PubMed]

33. Brzostek, A.; Dziadek, B.; Rumijowska-Galewicz, A.; Pawelczyk, J.; Dziadek, J. Cholesterol oxidase is required for virulence of Mycobacterium tuberculosis. FEMS Microbiol. Lett. 2007, 275, 106-112. [CrossRef] [PubMed]

34. Yang, X.; Gao, J.; Smith, I.; Dubnau, E.; Sampson, N.S. Cholesterol is not an essential source of nutrition for Mycobacterium tuberculosis during infection. J. Bacteriol. 2011, 193, 1473-1476. [CrossRef] [PubMed]

35. Brzostek, A.; Pawelczyk, J.; Rumijowska-Galewicz, A.; Dziadek, B.; Dziadek, J. Mycobacterium tuberculosis is able to accumulate and utilize cholesterol. J. Bacteriol. 2009, 191, 6584-6591. [CrossRef] [PubMed]

36. Petrusma, M.; van der Geize, R.; Dijkhuizen, L. 3-Ketosteroid $9 \alpha$-hydroxylase enzymes: Rieske non-heme monooxygenases essential for bacterial steroid degradation. Antonie Leeuwenhoek 2014, 106, 157-172. [CrossRef] [PubMed]

37. Hu, Y.; Van Der Geize, R.; Besra, G.S.; Gurcha, S.S.; Liu, A.; Rohde, M.; Singh, M.; Coates, A. 3-ketosteroid $9 \alpha$-hydroxylase is an essential factor in the pathogenesis of Mycobacterium tuberculosis. Mol. Microbiol. 2010, 75, 107-121. [CrossRef] [PubMed]

38. Rengarajan, J.; Bloom, B.R.; Rubin, E.J. Genome-wide requirements for Mycobacterium tuberculosis adaptation and survival in macrophages. Proc. Natl. Acad. Sci. USA 2005, 102, 8327-8332. [CrossRef] [PubMed]

39. Dresen, C.; Lin, L.Y.C.; D'Angelo, I.; Tocheva, E.I.; Strynadka, N.; Eltis, L.D. A flavin-dependent monooxygenase from Mycobacterium tuberculosis involved in cholesterol catabolism. J. Biol. Chem. 2010, 285, 22264-22275. [CrossRef] [PubMed]

40. Lack, N.A.; Yam, K.C.; Lowe, E.D.; Horsman, G.P.; Owen, R.L.; Sim, E.; Eltis, L.D. Characterization of a carbon-carbon hydrolase from Mycobacterium tuberculosis involved in cholesterol metabolism. J. Biol. Chem. 2010, 285, 434-443. [CrossRef] [PubMed]

41. Mukhopadhyay, S.; Nair, S.; Ghosh, S. Pathogenesis in tuberculosis: Transcriptomic approaches to unraveling virulence mechanisms and finding new drug targets. FEMS Microbiol. Rev. 2012, 36, 463-485. [CrossRef] [PubMed]

42. Van der Geize, R.; Grommen, A.W.F.; Hessels, G.I.; Jacobs, A.A.C.; Dijkhuizen, L. The steroid catabolic pathway of the intracellular pathogen Rhodococcus equi is important for pathogenesis and a target for vaccine development. PLoS Pathol. 2011, 7, e1002181. [CrossRef] [PubMed]

43. Driscoll, M.D.; McLean, K.J.; Levy, C.; Mast, N.; Pikuleva, I.A.; Lafite, P.; Rigby, S.E.; Leys, D.; Munro, A.W. Structural and biochemical characterization of Mycobacterium tuberculosis CYP142 evidence for multiple cholesterol 27-hydroxylase activities in a human pathogen. J. Biol. Chem. 2010, 285, 38270-38282. [CrossRef] [PubMed]

44. Marques, M.A.M.; Berrêdo-Pinho, M.; Rosa, T.L.; Pujari, V.; Lemes, R.M.; Lery, L.M.; Silva, C.A.M.; Guimarães, A.C.R.; Atella, G.C.; Wheat, W.H.; et al. The essential role of cholesterol metabolism in the intracellular survival of Mycobacterium leprae is not coupled to central carbon metabolism and energy production. J. Bacteriol. 2015, 197, 3698-3707. [CrossRef] [PubMed]

45. Guo, F.; Zhang, T.; Li, B.; Wang, Z.; Ju, F.; Liang, Y.-T. Mycobacterial species and their contribution to cholesterol degradation in wastewater treatment plants. Sci. Rep 2019, 9, 836. [CrossRef] [PubMed] 
46. Ventura, M.; Canchaya, C.; Tauch, A.; Chandra, G.; Fitzgerald, G.F.; Chater, K.F.; van Sinderen, D. Genomics of Actinobacteria: Tracing the evolutionary history of an ancient phylum. Microbiol. Mol. Biol. Rev. 2007, 71, 495-548. [CrossRef] [PubMed]

47. Parvez, M.; Qhanya, L.B.; Mthakathi, N.T.; Kgosiemang, I.K.R.; Bamal, H.D.; Pagadala, N.S.; Xie, T.; Yang, H.; Chen, H.; Theron, C.W.; et al. Molecular evolutionary dynamics of cytochrome P450 monooxygenases across kingdoms: Special focus on mycobacterial P450s. Sci. Rep. 2016, 6, 33099. [CrossRef] [PubMed]

48. Tortoli, E. Phylogeny of the genus Mycobacterium: Many doubts, few certainties. Infect. Genet. Evol. 2012, 12, 827-831. [CrossRef] [PubMed]

49. Mills, N. ChemDraw Ultra 10.0. J. Am. Chem. Soc. 2006, 128, 13649-13650. [CrossRef]

50. Marchler-Bauer, A.; Bo, Y.; Han, L.; He, J.; Lanczycki, C.J.; Lu, S.; Chitsaz, F.; Derbyshire, M.K.; Geer, R.C.; Gonzales, N.R.; et al. CDD/SPARCLE: Functional classification of proteins via subfamily domain architectures. Nucleic Acids Res. 2017, 45, D200-D203. [CrossRef] [PubMed]

51. Mthethwa, B.; Chen, W.; Ngwenya, M.; Kappo, A.; Syed, P.; Karpoormath, R.; Yu, J.H.; Nelson, D.; Syed, K. Comparative analyses of cytochrome P450s and those associated with secondary metabolism in Bacillus Species. Int. J. Mol. Sci. 2018, 19, 3623. [CrossRef] [PubMed]

52. Saeed, A.I.; Sharov, V.; White, J.; Li, J.; Liang, W.; Bhagabati, N.; Braisted, J.; Klapa, M.; Currier, T.; Thiagarajan, M.; et al. TM4: A free, open-source system for microarray data management and analysis. Biotechniques 2003, 34, 374-378. [CrossRef] [PubMed]

53. Kanehisa, M.; Furumichi, M.; Tanabe, M.; Sato, Y.; Morishima, K. KEGG: New perspectives on genomes, pathways, diseases and drugs. Nucleic Acids Res. 2017, 45, D353-D361. [CrossRef] [PubMed]

54. Cole, S.; Brosch, R.; Parkhill, J.; Garnier, T.; Churcher, C.; Harris, D.; Gordon, S.V.; Eiglmeier, K.; Gas, S.; Barry Iii, C.E.; et al. Deciphering the biology of Mycobacterium tuberculosis from the complete genome sequence. Nature 1998, 393, 537-544. [CrossRef] [PubMed]

55. Fleischmann, R.D.; Alland, D.; Eisen, J.A.; Carpenter, L.; White, O.; Peterson, J.; DeBoy, R.; Dodson, R.; Gwinn, M.; Haft, D.; et al. Whole-genome comparison of Mycobacterium tuberculosis clinical and laboratory strains. J. Bacteriol. 2002, 184, 5479-5490. [CrossRef] [PubMed]

56. Zheng, H.; Lu, L.; Wang, B.; Pu, S.; Zhang, X.; Zhu, G.; Shi, W.; Zhang, L.; Wang, H.; Wang, S.; et al. Genetic basis of virulence attenuation revealed by comparative genomic analysis of Mycobacterium tuberculosis strain H37Ra versus H37Rv. PLoS ONE 2008, 3, e2375. [CrossRef] [PubMed]

57. Madhavilatha, G.K.; Joseph, B.V.; Paul, L.K.; Kumar, R.A.; Hariharan, R.; Mundayoor, S. Whole-genome sequences of two clinical isolates of Mycobacterium tuberculosis from Kerala, South India. J. Bacteriol. 2012, 194, 4430. [CrossRef] [PubMed]

58. Zhang, Y.; Chen, C.; Liu, J.; Deng, H.; Pan, A.; Zhang, L.; Zhao, X.; Huang, M.; Lu, B.; Dong, H.; et al. Complete genome sequences of Mycobacterium tuberculosis strains CCDC5079 and CCDC5080, which belong to the Beijing family. J. Bacteriol. 2011, 193, 5591-5592. [CrossRef] [PubMed]

59. Tang, B.; Wang, Q.; Yang, M.; Xie, F.; Zhu, Y.; Zhuo, Y.; Wang, S.; Gao, H.; Ding, X.; Zhang, L.; et al. ContigScape: A Cytoscape plugin facilitating microbial genome gap closing. BMC Genom. 2013, 14, 289. [CrossRef] [PubMed]

60. Ilina, E.N.; Shitikov, E.A.; Ikryannikova, L.N.; Alekseev, D.G.; Kamashev, D.E.; Malakhova, M.V.; Parfenova, T.V.; Afanas'ev, M.V.; Ischenko, D.S.; Bazaleev, N.A.; et al. Comparative genomic analysis of Mycobacterium tuberculosis drug resistant strains from Russia. PLoS ONE 2013, 8, e56577. [CrossRef] [PubMed]

61. Isaza, J.P.; Duque, C.; Gomez, V.; Robledo, J.; Barrera, L.F.; Alzate, J.F. Whole genome shotgun sequencing of one Colombian clinical isolate of Mycobacterium tuberculosis reveals DosR regulon gene deletions. FEMS Microbiol. Lett. 2012, 330, 113-120. [CrossRef] [PubMed]

62. Miyoshi-Akiyama, T.; Matsumura, K.; Iwai, H.; Funatogawa, K.; Kirikae, T. Complete annotated genome sequence of Mycobacterium tuberculosis Erdman. J. Bacteriol. 2012, 194, 2770. [CrossRef] [PubMed]

63. Narayanan, S.; Deshpande, U. Whole-genome sequences of four clinical isolates of Mycobacterium tuberculosis from Tamil Nadu, south India. Genome Announc. 2013, 1, e00186-13. [CrossRef] [PubMed]

64. Roetzer, A.; Diel, R.; Kohl, T.A.; Rückert, C.; Nübel, U.; Blom, J.; Wirth, T.; Jaenicke, S.; Schuback, S.; Rüsch-Gerdes, S.; et al. Whole genome sequencing versus traditional genotyping for investigation of a Mycobacterium tuberculosis outbreak: A longitudinal molecular epidemiological study. PLoS Med. 2013, 10, e1001387. [CrossRef] [PubMed] 
65. Al Rashdi, A.S.A.; Jadhav, B.L.; Deshpande, T.; Deshpande, U. Whole-genome sequencing and annotation of a clinical isolate of Mycobacterium tuberculosis from Mumbai, India. Genome Announc. 2014, 2, e0154-14. [CrossRef] [PubMed]

66. Garnier, T.; Eiglmeier, K.; Camus, J.C.; Medina, N.; Mansoor, H.; Pryor, M.; Duthoy, S.; Grondin, S.; Lacroix, C.; Monsempe, C.; et al. The complete genome sequence of Mycobacterium bovis. Proc. Natl. Acad. Sci. USA 2003, 100, 7877-7882. [CrossRef] [PubMed]

67. Brosch, R.; Gordon, S.V.; Garnier, T.; Eiglmeier, K.; Frigui, W.; Valenti, P.; Dos Santos, S.; Duthoy, S.; Lacroix, C.; Garcia-Pelayo, C.; et al. Genome plasticity of BCG and impact on vaccine efficacy. Proc. Natl. Acad. Sci. USA 2007, 104, 5596-5601. [CrossRef] [PubMed]

68. Seki, M.; Honda, I.; Fujita, I.; Yano, I.; Yamamoto, S.; Koyama, A. Whole genome sequence analysis of Mycobacterium bovis bacillus Calmette-Guerin (BCG) Tokyo 172: A comparative study of BCG vaccine substrains. Vaccine 2009, 27, 1710-1716. [CrossRef] [PubMed]

69. Orduña, P.; Cevallos, M.A.; de León, S.P.; Arvizu, A.; Hernández-González, I.L.; Mendoza-Hernández, G.; López-Vidal, Y. Genomic and proteomic analyses of Mycobacterium bovis BCG Mexico 1931 reveal a diverse immunogenic repertoire against tuberculosis infection. BMC Genom. 2011, 12, 493. [CrossRef] [PubMed]

70. Joung, S.M.; Jeon, S.J.; Lim, Y.J.; Lim, J.S.; Choi, B.S.; Choi, I.Y.; Yu, J.H.; Na, K.I.; Cho, E.H.; Shin, S.S.; et al. Complete genome sequence of Mycobacterium bovis BCG Korea, the Korean vaccine strain for substantial production. Genome Announc. 2013, 1, e00069-13. [CrossRef] [PubMed]

71. Pan, Y.; Yang, X.; Duan, J.; Lu, N.; Leung, A.S.; Tran, V.; Hu, Y.; Wu, N.; Liu, D.; Wang, Z.; et al. The whole genome sequence of four BCG vaccine strains. J. Bacteriol. 2011, 193, 3152-3153. [CrossRef] [PubMed]

72. Bentley, S.D.; Comas, I.; Bryant, J.M.; Walker, D.; Smith, N.H.; Harris, S.R.; Thurston, S.; Gagneux, S.; Wood, J.; Antonio, M.; et al. The genome of Mycobacterium africanum West African 2 reveals a lineage-specific locus and genome erosion common to the M. tuberculosis complex. PLoS Negl. Trop. Dis. 2012, 6, e1552. [CrossRef] [PubMed]

73. Supply, P.; Marceau, M.; Mangenot, S.; Roche, D.; Rouanet, C.; Khanna, V.; Majlessi, L.; Criscuolo, A.; Tap, J.; Pawlik, A.; et al. Genomic analysis of smooth tubercle bacilli provides insights into ancestry and pathoadaptation of Mycobacterium tuberculosis. Nat. Genet. 2013, 45, 172. [CrossRef] [PubMed]

74. Cole, S.T.; Eiglmeier, K.; Parkhill, J.; James, K.D.; Thomson, N.R.; Wheeler, P.R.; Honore, N.; Garnier, T.; Churcher, C.; Harris, D.; et al. Massive gene decay in the leprosy bacillus. Nature 2001, 409, 1007. [CrossRef] [PubMed]

75. Monot, M.; Honoré, N.; Garnier, T.; Zidane, N.; Sherafi, D.; Paniz-Mondolfi, A.; Matsuoka, M.; Taylor, G.M.; Donoghue, H.D.; Bouwman, A.; et al. Comparative genomic and phylogeographic analysis of Mycobacterium leprae. Nat. Genet. 2009, 41, 1282-1289. [CrossRef] [PubMed]

76. Li, L.; Bannantine, J.P.; Zhang, Q.; Amonsin, A.; May, B.J.; Alt, D.; Banerji, N.; Kanjilal, S.; Kapur, V. The complete genome sequence of Mycobacterium avium subspecies paratuberculosis. Proc. Natl. Acad. Sci. USA 2005, 102, 12344-12349. [CrossRef] [PubMed]

77. Bannantine, J.P.; Li, L.; Mwangi, M.; Cote, R.; Garay, J.A.R.; Kapur, V. Complete genome sequence of Mycobacterium avium subsp. paratuberculosis, isolated from human breast milk. Genome Announc. 2014, 2, e01252-13. [CrossRef] [PubMed]

78. Amin, A.S.; Hsu, C.Y.; Darwish, S.F.; Ghosh, P.; AbdEl-Fatah, E.M.; Behour, T.S.; Talaat, A.M. Ecology and genomic features of infection with Mycobacterium avium subspecies paratuberculosis in Egypt. Microbiology 2015, 161, 807-818. [CrossRef] [PubMed]

79. Kim, B.J.; Choi, B.S.; Lim, J.S.; Choi, I.Y.; Lee, J.H.; Chun, J.; Kook, Y.H.; Kim, B.J. Complete genome sequence of Mycobacterium intracellulare clinical strain MOTT-02. J. Bacteriol. 2012, 194, 2771. [CrossRef] [PubMed]

80. Kim, B.J.; Choi, B.S.; Lim, J.S.; Choi, I.Y.; Kook, Y.H.; Kim, B.J. Complete genome sequence of Mycobacterium intracellulare clinical strain MOTT-64, belonging to the INT1 genotype. J. Bacteriol. 2012, 194, 3268. [CrossRef] [PubMed]

81. Kim, B.J.; Choi, B.S.; Lim, J.S.; Choi, I.Y.; Lee, J.H.; Chun, J.; Kook, Y.H.; Kim, B.J. Complete genome sequence of Mycobacterium intracellulare strain ATCC 13950T. J. Bacteriol. 2012, 194, 2750. [CrossRef] [PubMed]

82. Saini, V.; Raghuvanshi, S.; Khurana, J.P.; Ahmed, N.; Hasnain, S.E.; Tyagi, A.K.; Tyagi, A.K. Massive gene acquisitions in Mycobacterium indicus pranii provide a perspective on mycobacterial evolution. Nucleic Acids Res. 2012, 40, 10832-10850. [CrossRef] [PubMed] 
83. Kim, B.J.; Kim, B.R.; Lee, S.Y.; Seok, S.H.; Kook, Y.H.; Kim, B.J. Whole-genome sequence of a novel species, Mycobacterium yongonense DSM 45126T. Genome Announc. 2013, 1, e00604-13. [CrossRef] [PubMed]

84. Kim, B.J.; Choi, B.S.; Choi, I.Y.; Lee, J.H.; Chun, J.; Hong, S.H.; Kook, Y.H.; Kim, B.J. Complete genome sequence of Mycobacterium intracellulare clinical strain MOTT-36Y, belonging to the INT5 genotype. J. Bacteriol. 2012, 194, 4141-4142. [CrossRef] [PubMed]

85. Gallien, S.; Perrodou, E.; Carapito, C.; Deshayes, C.; Reyrat, J.M.; Van Dorsselaer, A.; Poch, O.; Schaeffer, C.; Lecompte, O. Ortho-proteogenomics: Multiple proteomes investigation through orthology and a new MS-based protocol. Genome Res. 2009, 19, 128-135. [CrossRef] [PubMed]

86. Mohan, A.; Padiadpu, J.; Baloni, P.; Chandra, N. Complete genome sequences of a Mycobacterium smegmatis laboratory strain (MC2 155) and isoniazid-resistant (4XR1/R2) mutant strains. Genome Announc. 2015, 3, e01520-14. [CrossRef] [PubMed]

87. Kallimanis, A.; Karabika, E.; Mavromatis, K.; Lapidus, A.; LaButti, K.M.; Liolios, K.; Ivanova, N.; Goodwin, L.; Woyke, T.; Velentzas, A.D.; et al. Complete genome sequence of Mycobacterium sp. strain (Spyr1) and reclassification to Mycobacterium gilvum Spyr1. Stand. Genom. Sci. 2011, 5, 144. [CrossRef] [PubMed]

88. Bragin, E.Y.; Shtratnikova, V.Y.; Dovbnya, D.V.; Schelkunov, M.I.; Pekov, Y.A.; Malakho, S.G.; Egorova, O.V.; Ivashina, T.V.; Sokolov, S.L.; Ashapkin, V.V.; et al. Comparative analysis of genes encoding key steroid core oxidation enzymes in fast-growing Mycobacterium spp. strains. J. Steroid Biochem. Mol. Biol. 2013, 138, 41-53. [CrossRef] [PubMed]

89. Kato, H.; Ogawa, N.; Ohtsubo, Y.; Oshima, K.; Toyoda, A.; Mori, H.; Nagata, Y.; Kurokawa, K.; Hattori, M.; Fujiyama, A.; et al. Complete genome sequence of a phenanthrene degrader, Mycobacterium sp. strain EPa45 (NBRC 110737), isolated from a phenanthrene-degrading consortium. Genome Announc. 2015, 3, e00782-15. [CrossRef] [PubMed]

90. Yu, B.; Tao, F.; Li, F.; Hou, J.; Tang, H.; Ma, C.; Xu, P. Complete genome sequence of Mycobacterium goodii X7B, a facultative thermophilic biodesulfurizing bacterium with industrial potential. J. Biotechnol. 2015, 212, 56-57. [CrossRef] [PubMed]

91. Costa, K.C.; Bergkessel, M.; Saunders, S.; Korlach, J.; Newman, D.K. Enzymatic degradation of phenazines can generate energy and protect sensitive organisms from toxicity. mBio 2015, 6, e01520-15. [CrossRef] [PubMed]

92. Stinear, T.P.; Seemann, T.; Pidot, S.; Frigui, W.; Reysset, G.; Garnier, T.; Meurice, G.; Simon, D.; Bouchier, C.; Ma, L.; et al. Reductive evolution and niche adaptation inferred from the genome of Mycobacterium ulcerans, the causative agent of Buruli ulcer. Genome Res. 2007, 17, 192-200. [CrossRef] [PubMed]

93. Zhang, Z.Y.; Sun, Z.Q.; Wang, Z.L.; Wen, Z.L.; Sun, Q.W.; Zhu, Z.Q.; Song, Y.Z.; Zhao, J.W.; Wang, H.H.; Zhang, S.L.; et al. Complete genome sequence of a novel clinical isolate, the nontuberculous Mycobacterium strain JDM601. J. Bacteriol. 2011, 193, 4300-4301. [CrossRef] [PubMed]

94. Stinear, T.P.; Seemann, T.; Harrison, P.F.; Jenkin, G.A.; Davies, J.K.; Johnson, P.D.; Abdellah, Z.; Arrowsmith, C.; Chillingworth, T.; Churcher, C.; et al. Insights from the complete genome sequence of Mycobacterium marinum on the evolution of Mycobacterium tuberculosis. Genome Res. 2008, 18, 729-741. [CrossRef] [PubMed]

95. Tobias, N.J.; Doig, K.D.; Medema, M.H.; Chen, H.; Haring, V.; Moore, R.; Seemann, T.; Stinear, T.P. Complete genome sequence of the frog pathogen Mycobacterium ulcerans ecovar Liflandii. J. Bacteriol. 2013, 195, 556-564. [CrossRef] [PubMed]

96. Tufariello, J.M.; Kerantzas, C.A.; Vilchèze, C.; Calder, R.B.; Nordberg, E.K.; Fischer, J.A.; Hartman, T.E.; Yang, E.; Driscoll, T.; Cole, L.E.; et al. The complete genome sequence of the emerging pathogen Mycobacterium haemophilum explains its unique culture requirements. mBio 2015, 6, e01313-15. [CrossRef] [PubMed]

97. Ripoll, F.; Pasek, S.; Schenowitz, C.; Dossat, C.; Barbe, V.; Rottman, M.; Macheras, E.; Heym, B.; Herrmann, J.L.; Daffé, M.; et al. Non mycobacterial virulence genes in the genome of the emerging pathogen Mycobacterium abscessus. PLoS ONE 2009, 4, e5660. [CrossRef] [PubMed]

98. Kim, B.J.; Kim, B.R.; Hong, S.H.; Seok, S.H.; Kook, Y.H.; Kim, B.J. Complete genome sequence of Mycobacterium massiliense clinical strain Asan 50594, belonging to the type II genotype. Genome Announc. 2013, 1, e00429-13. [CrossRef] [PubMed] 
99. Raiol, T.; Ribeiro, G.M.; Maranhão, A.Q.; Bocca, A.L.; Silva-Pereira, I.; Junqueira-Kipnis, A.P.; de Macedo Brigido, M.; Kipnis, A. Complete genome sequence of Mycobacterium massiliense. J. Bacteriol. $2012,194$. [CrossRef] [PubMed]

100. Sekizuka, T.; Kai, M.; Nakanaga, K.; Nakata, N.; Kazumi, Y.; Maeda, S.; Makino, M.; Hoshino, Y.; Kuroda, M. Complete genome sequence and comparative genomic analysis of Mycobacterium massiliense JCM 15300 in the Mycobacterium abscessus group reveal a conserved genomic island MmGI-1 related to putative lipid metabolism. PLoS ONE 2014, 9, e114848. [CrossRef] [PubMed] 